\title{
Violet stimulated luminescence dating of quartz from Luochuan (Chinese loess plateau): Agreement with independent chronology up to $600 \mathrm{ka}$
}

Ankjærgaard, C.; Guralnik, B.; Buylaert, Jan-Pieter; Reimann, T.; Yi, S. W.; Wallinga, J.

Published in:

Quaternary Geochronology

Link to article, DOI:

10.1016/j.quageo.2016.03.001

Publication date:

2016

Document Version

Peer reviewed version

Link back to DTU Orbit

Citation (APA):

Ankjærgaard, C., Guralnik, B., Buylaert, J-P., Reimann, T., Yi, S. W., \& Wallinga, J. (2016). Violet stimulated luminescence dating of quartz from Luochuan (Chinese loess plateau): Agreement with independent chronology up to 600 ka. Quaternary Geochronology, 34, 33-46. https://doi.org/10.1016/j.quageo.2016.03.001

\section{General rights}

Copyright and moral rights for the publications made accessible in the public portal are retained by the authors and/or other copyright owners and it is a condition of accessing publications that users recognise and abide by the legal requirements associated with these rights.

- Users may download and print one copy of any publication from the public portal for the purpose of private study or research.

- You may not further distribute the material or use it for any profit-making activity or commercial gain

- You may freely distribute the URL identifying the publication in the public portal 


\section{Violet stimulated luminescence dating of quartz from Luochuan (Chinese} Loess Plateau): agreement with independent chronology up to 600 ka.

C. Ankjærgaard ${ }^{1, *}$, B. Guralnik ${ }^{1}$, J.-P. Buylaert ${ }^{2,3}$, T. Reimann ${ }^{1}$, S.W. Yi ${ }^{4}$, J. Wallinga $^{1}$

${ }^{1}$ Soil Geography and Landscape group \& Netherlands Centre for Luminescence dating, Wageningen University, PO Box 47, 6700 AA Wageningen, The Netherlands

${ }^{2}$ Nordic Laboratory for Luminescence Dating, Department of Geoscience, Aarhus University, Ris $\varnothing$ Campus, DK-4000 Roskilde, Denmark

${ }^{3}$ Centre for Nuclear Technologies, Technical University of Denmark, Risø Campus, DK4000 Roskilde, Denmark

${ }^{4}$ School of Geographic and Oceanographic Sciences, Nanjing University, China

* Corresponding author: christina.ankjaergaard@gmail.com

\section{Keywords}

Violet Stimulated Luminescence (VSL), natural dose response curve, Chinese loess plateau, Luochuan, luminescence dating, Quaternary

\footnotetext{
Abstract

Luminescence dating at the Luochuan loess type section (China) section is at present limited to $\sim 0.1$ Ma using quartz blue light stimulated luminescence (BLSL), but can be extended back in time to $\sim 0.5 \mathrm{Ma}$ by resorting to the more developmental post-infrared infrared stimulated luminescence (post-IR IRSL) and thermally transferred OSL (TT-OSL) signals. Since both the latter are associated with systematic uncertainties due to the potential (a)-thermal instability of these signals, a search continues for alternative, and demonstrably stable luminescence signals that can cover the entire Quaternary timescale. Here we explore the violet stimulated luminescence (VSL) signal at the Luochuan section, which provides a continuous archive of homogenous sediment with favourable luminescence characteristics and a solid independent age framework. By testing several luminescence protocols and their associated performance, we demonstrate that the Multi-Aliquot Additive-Dose (MAAD) protocol produces a VSL chronology at Luochuan which is in agreement with independent ages up to $\sim 0.6 \mathrm{Ma}$. For a more representative environmental dose rate of $\sim 2 \mathrm{~Gy} / \mathrm{ka}(\sim 35 \%$ lower than at Luochuan), the documented range of MAAD-VSL sensitivity (200-1800 Gy)
} 
would correspond to the ability to date sediment up to $\sim 1$ Ma back in time, offering a remarkable advance over existing methods.

\section{Introduction}

Quartz and feldspar are the two most widely used minerals in optically stimulated luminescence (OSL) dating, a method crucial for establishing an absolute chronology for terrestrial sedimentary sequences during the Quaternary (e.g. Preusser et al., 2009). The OSL method determines the time since sediments were last exposed to sunlight (usually the time of deposition), and is based on quantification of the environmental ionizing radiation dose that a mineral absorbs during its burial when shielded from sunlight. The upper OSL dating limit is defined by the threshold dose beyond which the luminescence signal no longer increases with burial time. The dose at which the luminescence signal is considered to become insensitive to further increase in absorbed dose, has traditionally been set at twice the characteristic dose, $\mathrm{D}_{0}$ (Gy) (Wintle and Murray, 2006), or 15\% short of the signal's steady state in nature (Guralnik et al., 2015). This typically limits the quartz fast component blue OSL (BLSL) to less than $200 \mathrm{~Gy}$, resulting in a quartz dating limit between 100-200 ka for typical environmental dose rates in sedimentary environments (Wintle and Murray 2006; Wintle, 2008). Feldspar OSL signals have a later saturation at about $\sim 1 \mathrm{kGy}$, but are usually associated with $\sim 30 \%$ higher dose rates, resulting in an upper dating limit of between 300-600 ka (Buylaert et al., 2012; Li and Li, 2012; Kars et al., 2012; Joordens et al., 2015). For both quartz and feldspar, the specific environment determines the applicability of the dating method and its limitations, and although feldspar possesses the characteristics to date older samples compared to quartz BLSL, the signal is not entirely stable, which might lead to systematic age underestimations if not properly corrected for (e.g. Li et al., 2008; Kars et al., 2012).

In the quest for extending the upper age range of luminescence dating, different methods using both quartz (Singarayer et al. 2000; Jain et al. 2005; Duller and Wintle, 2012) and feldspar (Buylaert et al., 2012; Li and Li, 2012; Li et al., 2014) have been proposed. One method using quartz was initially proposed by Jain (2009), who used violet stimulation (405 $\mathrm{nm}$ ) to measure the luminescence (abbreviated VSL) from a deep electron trap in quartz not accessible by blue light. Jain (2009) reported VSL $\mathrm{D}_{0}$ values from quartz that were 5-15 times larger than those of BLSL. It was subsequently confirmed, that the origin of the VSL signal is associated with a thermoluminescence (TL) electron trap at $\sim 380^{\circ} \mathrm{C}$ (Ankjærgaard et al. 2013; Hernandez and Mercier, 2015), with a thermal stability of $\sim 10^{11}$ years at $10^{\circ} \mathrm{C}$ 
(Ankjærgaard et al. 2013; 2015). Additionally, extended fading experiments indicate no observable athermal loss (Ankjærgaard et al. 2013). These findings indicate negligible thermal and athermal losses of the VSL signal on the timescale of interest ( $10^{6}$ years). Attempts to validate quartz VSL dating have not all been successful. VSL ages of sediments were found in agreement with BLSL ages on a timescale $<0.3 \mathrm{Ma}$ for a sedimentary core from the southern Netherlands ( $n=9$; Ankjærgaard et al., 2013). However, the dating of a palaeosol sequence in Israel, capped by known-age (K-Ar) basalts $(1.58-0.96 \mathrm{Ma})$, proved to be more challenging, exhibiting age underestimations of $\sim 50 \%$ or more (Ankjærgaard et al., 2015). Although this underestimation may be related to the thermal history of that site, another possible explanation is the potential inability of the current VSL protocol to successfully convert natural signals into palaeodoses; i.e. that the signal growth in nature cannot be accurately reproduced in the laboratory.

In this paper, we investigate the similarity in shape between the natural VSL growth and that obtained in the laboratory based on quartz samples from the Luochuan section on the Chinese loess plateau. The loess plateau has been steadily accumulating windblown dust throughout the last 2.6 Ma (Liu, 1985) and can therefore be used as a natural laboratory for luminescence signal growth during the Quaternary (e.g. Lai, 2010; Chapot et al., 2012; Li and Li, 2012; Chapot et al., in press). Due to the constant influx of dust from the same provenance, the variation in environmental dose rates and luminescence behaviour is minimal, causing the main variable throughout the section to be the accumulated natural dose. The expected natural doses can be established using the chronology of palaeosol/loess transitions (Lu et al., 1999; Ding et al., 2002); these ages are based on correlation of grain size records from five loess sections throughout the Chinese loess plateau with the variations in the Earth's obliquity and precession as well as magnetic susceptibility and deep sea $\delta^{18} \mathrm{O}$ records. Due to the high resolution of independently estimated ages throughout the Luochuan section, samples obtained from this site can be used to construct a 'natural' dose response curve (DRC) where the duration of exposure to environmental radiation results in increasing absorbed dose down the profile. The absorbed dose is calculated from the age of the deposits (Ding et al., 2002) in combination with the environmental dose rate as determined by gamma spectrometry for each of the samples. The natural DRC can then be compared to laboratory irradiated DRCs from the same material to test their similarity. This approach has so far been attempted on loess sequences by a few studies only (Lai, 2010; Chapot et al., 2012; Li and Li, 2012; TimarGabor and Wintle, 2013; Chapot et al., in press) demonstrating for all methods investigated (quartz BLSL; thermally transferred OSL, TT-OSL; feldspar post-infrared infrared stimulated 
luminescence, post-IR IRSL), that the natural and laboratory-regenerated DRCs start to diverge in shape after a certain dose, thereby causing a progressive underestimation of ages at all larger doses.

Specifically for the Luochuan loess section, the deviation between natural and laboratory DRCs has been investigated using BLSL, TT-OSL and IRSL, as discussed below.

(i) Age underestimations for quartz BLSL at Luochuan was observed at the L1/S1 boundary (MIS5/4 transition) by Buylaert et al. (2007), suggesting a maximum age limit at about 40-50 ka, corresponding to a maximum $\mathrm{D}_{\mathrm{e}}$ of about 120-150 Gy. Lai (2010), using 20 samples in the range $75-3000 \mathrm{~Gy}$, also reported dose underestimations for quartz BLSL starting from 230 Gy compared to the loesspalaeosol sequence, but for DRCs fitted with saturating exponential-plus-linear functions, contrary to Buylaert et al. (2007), where the DRCs were fitted with single exponentials. Chapot et al. (2012) investigated the onset of deviation between natural and SAR DRCs using seven samples with doses in the range $40-$ 2000 Gy. They estimated the deviation to commence around 150 Gy when fitting the DRCs with a single or double saturating exponential. These studies highlight that even though it is possible to measure quartz BLSL laboratory regenerated DRCs beyond several hundred Gy, the natural signals progressively deviate from the laboratory trend beyond $\sim 150 \mathrm{~Gy}$, setting an upper applicability limit for accurate quartz BLSL dating of $\sim 50 \mathrm{ka}$ at this site (Buylaert et al., 2007; Lai, 2010; Chapot et al., 2012).

(ii) Quartz TT-OSL is one of the main promising methods to extend the luminescence dating age range. Four samples taken close to the Brunhes/Matuyama (B/M) boundary at the Luochuan type section gave an average age of $771 \pm 15$ in full agreement with its independent age of $775.6 \pm 1.9 \mathrm{ka}$ (Wang et al., 2006, and references therein). Although this date is very encouraging, the thermal stability of the TT-OSL signal in this age range has been extensively debated and research suggests that a thermal correction is needed to adjust for charge loss (e.g. Adamiec et al., 2010; Duller and Wintle, 2012, Thiel et al., 2012). Recently Chapot et al. (in press) demonstrated using 21 samples in the range $0-4300 \mathrm{~Gy}$, that although the natural TT-OSL DRC saturates only at $\sim 2200 \mathrm{~Gy}$, it starts to deviate from the laboratory TT-OSL DRCs already at $\sim 150 \mathrm{~Gy}$, causing large age underestimates beyond $\sim 50 \mathrm{ka}$. Nevertheless, using an estimate of the thermal lifetime of the signal, Chapot et al. (in press) raise a possibility to correct the TT-OSL ages, 
obtaining a meaningful chronology up until $\sim 500 \mathrm{ka}$. It thus remains a possibility that the B/M age obtained by quartz TT-OSL in Wang et al. (2006) is a fortuitous coincidence rather than a methodologically-consistent result (Chapot et al., 2014).

(iii) For feldspar post-IR IRSL signals, Li and Li (2012) showed that the 250 and 300 ${ }^{\circ} \mathrm{C}$ signals in the multi-elevated-temperature (MET-pIRIR) protocol resulted in correct ages up to $\sim 300 \mathrm{ka}$ (equivalent to a dose limit of $\sim 900 \mathrm{~Gy}$ ) using thirteen samples with natural predicted doses between 70 and $1200 \mathrm{~Gy}$. This limit was further extended to $470 \mathrm{ka}$ by a pre-dose MET-pIRIR SAR protocol (Li et al., 2014), utilising the observation that the test-dose signal can retain a 'memory' of the dose prior received in nature. The latter was demonstrated on 7 samples (including three from the Luochuan section), spanning expected doses between 100 and $1600 \mathrm{~Gy}$.

Although the dating limit of standard quartz BLSL at this site ( $50 \mathrm{ka})$ appears to have been superseded by feldspar pre-dose MET-pIRIR ( 470 ka) and quartz TT-OSL ( 500 ka), these latter approaches are based on potentially unstable signals, which may fade over geological time through loss of signal due to either tunnelling or thermal recombination of trapped charge, respectively ( $\mathrm{Li}$ and $\mathrm{Li}, 2012$; Chapot et al., in press). From a formal propagation of uncertainties, it may be shown that such ages, when obtained in the 0.1-0.5 Ma timespan, are often too close to the saturation limit to allow robust equivalent dose estimation. Although this problem can be circumvented by presenting "minimum age estimates" only (e.g. Joordens et al., 2015), there is a clear need for alternative, and demonstrably stablesignal approaches.

In this work, we investigate 24 quartz samples from the Luochuan site with depositional ages in the range 11-2500 ka (Ding et al., 2002) with the aim of studying the similarity in shape between the natural VSL growth with that obtained upon beta-irradiation in the laboratory. Two different methods are tested, the single aliquot regenerative dose (SAR) and the multiple-aliquot additive-dose (MAAD) protocols, and the success of the two protocols is evaluated against independent age control to infer the maximum limit of VSL dating at this site. 


\section{Methods and materials}

\subsection{Instrumentation}

Optical stimulation was done on a Ris $\varnothing$ TL/OSL-20 reader, adapted for stimulation with both blue LEDs ( $470 \pm 30 \mathrm{~nm} ; 54 \mathrm{~mW}$ at sample position) and a violet solid-state laser diode (405 nm; $35 \mathrm{~mW}$ at sample position). The focused Nichia $405 \mathrm{~nm}$ laser diode gave a uniform illumination area of $\sim 3.5 \mathrm{~mm}$ in diameter, effectively limiting all sample diameters to $3 \mathrm{~mm}$ mask size on stainless steel discs. To cut off the short wavelength tail produced by the violet laser diode, a combination of a laser bandpass filter $402 \pm 15 \mathrm{~nm}$ (AHF F49-402) and a 3-mm-thick ITOS GG395 glass filter was inserted in front of the laser diode. Luminescence was recorded using a photomultiplier tube (EMI QA 9235), equipped with a 7.5mm HoyaU340 filter, and a Semrock Brightline $340 \mathrm{~nm}$ interference filter (FF01-340/26), the latter to minimize violet laser breakthrough into the photomultiplier tube. Samples were irradiated using a ${ }^{90} \mathrm{Sr} /{ }^{90} \mathrm{Y}$ source incorporated in the reader, with a dose rate of $0.18 \pm 0.01 \mathrm{~Gy} \mathrm{~s}^{-1}$.

\subsection{Sample site, independent age constraints and expected natural doses}

For this study, a total of 27 luminescence samples were collected from the Luochuan type section. Of these, twenty-four samples (codes 148101-24) were taken from loess (L) and palaeosol layers (S) from the Holocene soil S0 down to palaeosol S6, and one sample from the loess just above the 2.6 Ma loess -red clay boundary (sample 148125). Two additional samples were collected from L9 (128187), and from just above the 2.6 Ma loess -red clay boundary (sample 128188; note that the latter is from the same unit as 148125 but from a different location). Of these 27 samples, three yielded very little quartz $(148103,148116$, 148120) and have not been considered further. An overview of the final 24 samples used to construct the natural VSL dose response curve is provided in Table 1.

The independent ages arise from a linear interpolation between the stable-isotope chronology of Ding et al. (2002), with an assigned conservative uncertainty of $10 \%$. For the upper two samples (148101-02), we measured multi-grain quartz BLSL D $\mathrm{D}_{\mathrm{e}}$ values (preheat: $260{ }^{\circ} \mathrm{C}$ for $10 \mathrm{~s}$ and cut-heat: $220{ }^{\circ} \mathrm{C}$; signal:0-0.32 s and background: 0.32-0.64 s; Table 1). Sample-specific environmental dose rates were determined as described in the section below. The expected natural doses in these quartz samples (Table 1) were estimated by multiplying the independent age of each sample by its measured environmental dose rate, except for samples 148101 and -02 for which the quartz SAR BLSL $D_{e}$ values are used. 


\subsection{Sample preparation and environmental dose rate determination}

Samples were collected in the field by S.W. Yi in 2014, by hammering stainless steel tubes (diameter: $5 \mathrm{~cm}$, length: $30 \mathrm{~cm}$ ) into freshly cleaned loess exposures at Luochuan. The material at the ends of each tube ( $250 \mathrm{~g}$ dry sediment) was removed under dark room conditions and used for dose rate determination. The inner material was not exposed to light during sampling, and hence suitable for luminescence analysis. This material was wet-sieved to extract the $63-90 \mu \mathrm{m}$ grain-size fraction which was then treated with $10 \% \mathrm{HCl}$ and $10 \%$ $\mathrm{H}_{2} \mathrm{O}_{2}$ to remove carbonate and organic matter, respectively. This fraction was subsequently etched for 20 min using 10\% HF to remove clay and iron oxide coatings. In between each chemical treatment step the fraction was washed multiple times with distilled water. After drying at $50^{\circ} \mathrm{C}$, a heavy liquid separation at $2.58 \mathrm{~g} / \mathrm{cm}^{3}$ was carried out to remove the K-rich feldspar fraction. The heavier quartz-rich fraction was then etched for 60 mins using concentrated HF (35\%) to remove remaining feldspars and etch away the outer alphairradiated layer. Following etching, the quartz fraction was treated with $10 \% \mathrm{HCl}$ for 60 mins to remove any possible precipitated fluorides and washed several times with distilled water before drying at $50^{\circ} \mathrm{C}$. The purity of the quartz extract was confirmed by the absence of a significant IRSL signal (ratio of IRSL to OSL signal <2\%). For VSL measurements, the quartz grains were mounted on stainless-steel discs using silicon spray as an adhesive, limiting the sample diameter to $3 \mathrm{~mm}$ due to the laser spot size (see Section 2.1).

For the environmental dose rate determination, the material was air-dried, ashed at $450^{\circ} \mathrm{C}$ for $24 \mathrm{~h}$ and homogenised before mixing with wax to produce a gamma cup. These cups were stored for $\sim 3$ weeks to allow ${ }^{222} \mathrm{Rn}$ to build up an equilibrium with ${ }^{226} \mathrm{Rn}$ before inserted into a high resolution gamma spectrometer (calibrated according to Murray et al., 1987) and counted for $\sim 24 \mathrm{~h}$. Radionuclide concentrations are given in Table 1 and these values were converted to dry beta and gamma dose rates using the conversion factors given in Guérin et al. (2011). Average water content of $15 \pm 5 \%$ and $20 \pm 5 \%$ was assumed for the loess and palaeosols respectively ( $\mathrm{Li}$ and $\mathrm{Li}, 2012$ ). An internal quartz dose rate of $0.02 \pm 0.01 \mathrm{~Gy} / \mathrm{ka}$ (Vandenberghe et al., 2007) and a contribution from cosmic rays (Prescott and Hutton, 1994) were also incorporated into the total dose rate. The total environmental dose rates are listed in Table 1.

\section{Natural decay and dose response}

The natural VSL signals from the 24 samples listed in Table 1 have been measured using the protocol given in Table 2. The approach is similar to that used in Ankjærgaard et al., 
(2015) with the main differences being (i) a test dose preheat of $300{ }^{\circ} \mathrm{C}$ (rather than $290{ }^{\circ} \mathrm{C}$ ) for $100 \mathrm{~s}$, i.e. identical to the regenerative dose preheat such that the exact same VSL signal is measured, and (ii) the signal clean-out (at the end of each measurement cycle) is a TL to 500 ${ }^{\circ} \mathrm{C}$ (sample held at $500{ }^{\circ} \mathrm{C}$ for $20 \mathrm{~s}$ ) instead of a VSL bleach at $380{ }^{\circ} \mathrm{C}$ for $200 \mathrm{~s}$, as this showed the lowest residual signal (data not presented).

The natural $\left(\mathrm{L}_{\mathrm{n}}\right)$ and test dose $\left(\mathrm{T}_{\mathrm{n}}\right)$ luminescence signals were measured for six aliquots of each sample in Table 1. Figure 1a shows the absolute intensity and signal decay shape for six representative samples with expected natural doses spanning between 60 and 7900 Gy. Given the rather dim nature of the VSL signals in Fig. 1a, we adopted signal summation across all aliquots, to maximize the signal to noise ratio. Furthermore, to enable direct comparison of intensity between the samples, the summed decay curves were normalised to the initial intensity (first $0.5 \mathrm{~s}$ ) of their corresponding test dose (540 Gy) measurements (Fig. 1b), which should cancel out differences in luminescence intensity due to variable sample mass. Although the dynamic range of signal intensities is not very large, Fig. $1 \mathrm{~b}$ shows that the normalised VSL intensities increase with dose, over a range of several kGy.

We constructed a natural dose response curve (DRC) for the 24 samples at the Luochuan site, by plotting the average $\mathrm{L}_{\mathrm{n}} / \mathrm{T}_{\mathrm{n}}$ of each sample, as a function of its independently estimated dose (Fig. 2). In order to reduce intra-sample scatter, and given the limited number of aliquots measured per sample $(n=6)$, the reported average $\mathrm{L}_{\mathrm{n}} / \mathrm{T}_{\mathrm{n}}$ values were calculated after removal of individual $\mathrm{L}_{\mathrm{n}} / \mathrm{T}_{\mathrm{n}}$ outliers according to the standard procedure employed in box-plots (Chambers et al., 1983; p. 62), excluding at most two observation per sample (i.e. $<33 \%$ of data). Such outlier removal was justified through a complementary experiment, in which natural signals were measured for 48 additional aliquots of sample 148109 (resulting in a total of $n=54$ realisations). After an identical removal of outliers, the mean and the standard error of the small dataset $\left(\mathrm{L}_{\mathrm{n}} / \mathrm{T}_{\mathrm{n}}=0.76 \pm 0.03 ; n=5\right)$ was found within error of the extended dataset $\left(\mathrm{L}_{\mathrm{n}} / \mathrm{T}_{\mathrm{n}}=0.74 \pm 0.01 ; n=47 ; \mathrm{p}=0.01\right.$ for a normal distribution $)$. The latter check demonstrates that $n=4-6$ observations of $\mathrm{L}_{n} / \mathrm{T}_{\mathrm{n}}$ per sample are acceptably representative.

The VSL natural DRC for the Luochuan loess site (Fig. 2), shows that although the relationship between the natural VSL signal and dose remains noisy even after data reduction, there is a clear correlation between $\mathrm{L}_{\mathrm{n}} / \mathrm{T}_{\mathrm{n}}$ and $\log _{10}(\mathrm{dose})$ (Pearson's $r=0.88$ ), implying a strong functional dependence of luminescence on dose. With 24 samples, this demonstrates, for the first time, that the natural VSL signal of quartz is a dosimeter in the natural environment in the 200 - 2000 Gy range, and thereby of interest for dating about tenfold beyond the accepted limit of quartz BLSL dating. 
In Fig. 2, the data has been fitted (adjusted $R^{2}=0.81$ ) with a single saturating exponential with a constant vertical offset of $0.45 \pm 0.05$ and a characteristic dose $\mathrm{D}_{0}$ of $921 \pm$ $222 \mathrm{~Gy}$. The offset appears to be an artefact of the sampled dose domain (with only two samples with expected doses $<200$ Gy). Furthermore, such an offset is analogous to fitting VSL growth curves with a single saturating exponential with an X-axis offset (Jain, 2009; Hernandez and Mercier, 2015), and represents a simplified case of fitting a sum of two saturating exponentials (Ankjærgaard et al., 2013; 2015), thereby fully in line with previous research.

Although the true shape of the natural growth at low doses is important - especially for comparison between BLSL and VSL ages, from a dating point of view, the added value of the VSL signal is for dating at high doses when the OSL signal is in saturation which for this site occurs beyond 150 Gy (Buylaert et al., 2007; Chapot et al., 2012). It is therefore remarkable, that the natural VSL signal exhibits saturation only at doses exceeding $~ 1800 \mathrm{~Gy}$ (Fig. 2), which would potentially allow dating of the Luochuan loess section using VSL up to $600 \mathrm{ka}$.

\section{Single-aliquot regenerative (SAR) dose response}

\subsection{Natural vs. regenerated decay curves}

Reported VSL dose-response curves (Jain, 2009; Ankjærgaard et al., 2013; 2015; Hernandez and Mercier, 2015) have so far all been measured using different variations of the single-aliquot regenerative (SAR) dose protocol (Murray and Wintle, 2003; Wintle and Murray, 2006) originally developed for quartz BLSL dating. All VSL protocols (including the one used here, Table 2), are based on measuring either the natural or a regenerated violet luminescence signal, after the removal of the BLSL signal (by blue light stimulation at some elevated temperature). In this study, SAR VSL dose response curves were measured for a single aliquot for six representative samples (natural doses spanning 45-7900 Gy). All six aliquots, which were previously used for constructing the natural DRC (Fig. 2), were irradiated with approximately geometrically progressing doses of $0,15,30,60,115,230,460$, 920, 1840, 3680, and $8100 \mathrm{~Gy}$, using the protocol in Table 2 with a test dose of $540 \mathrm{~Gy}$. A recycling regeneration dose of $230 \mathrm{~Gy}$ was added at the end of the sequence, confirming $\mathrm{L}_{\mathrm{x}} / \mathrm{T}_{\mathrm{x}}$ reproducibility (per fixed dose) within $10 \%$.

Figure 3 compares the natural and regenerated VSL decay curves for four representative of the abovementioned six samples. Note that although the samples were stimulated by violet light for $500 \mathrm{~s}$, only the first $100 \mathrm{~s}$ of VSL signals are shown for a better 
visualisation of the change in shape in this initial part of the signal. Two emergent observations, valid for all samples (also those not shown in Fig. 3) are: (i) the regenerated decay curve signals become progressively slower with increasing regeneration doses, and (ii) the shape of the natural decay curve is markedly different from that of the regenerated decays, although least pronounced for the sample with the highest natural dose of $7900 \mathrm{~Gy}$ (see Fig. $3 d)$.

Interpolation of a natural signal onto a laboratory-regenerated DRC implicitly assumes that the natural and regenerated decay curves are not dissimilar in shape, as a marked difference would imply sensitivity changes imposed by the measurement protocol. This assumption is not met for the natural and regenerated VSL decays in Fig. 3, due to the dissimilarity of their respective decay shapes, and further shape change as a function of irradiation dose for the regenerative decay. The dependence of VSL decay shape on dose has been previously attributed to non-first order kinetics (Ankjærgaard et al., 2013), although the retrapping mechanisms have not been investigated since. While Hernandez and Mercier (2015) adopted a breakdown of VSL decay into three exponential components plus a constant offset, such a breakdown has to be justified by an evaluation of (i) residuals and (ii) the tradeoffs between reducing the residuals at the expense of adding more model parameters.

Unlike simple signal integration, multi-exponential fitting may enable the extraction of a common component in signals with markedly different shapes, if such a component can be shown to exist and if it is separable. For signal extraction, this would circumvent the difference in shape between the natural and regenerated decay curves and allow the comparison of the same component across different signals. To test the approach of Hernandez and Mercier (2015) who devise three exponential components, we fitted all our natural and regenerated decay curves with:

$L_{\mathrm{VSL}}(t)=a_{1} \cdot \exp \left(-t / \tau_{1}\right)+a_{2} \cdot \exp \left(-t / \tau_{2}\right)+a_{3} \cdot \exp \left(-t / \tau_{3}\right)+c$, where $a_{i}$ [a.u.] is the initial intensity of the $i$-th component, $\tau_{i}[\mathrm{~s}]$ is its corresponding lifetime and $c$ [a.u.] the background counts possibly including a very slow decaying signal. Assuming that the natural and all subsequent regenerated curves for each sample contain varying intensities of the same (fixed) exponential components, we performed a simultaneous global fit of the natural and all subsequent regenerative decay curves, with $\tau_{1^{-}} \tau_{3}$ fixed for each sample. Failing to obtain plausible fits, we relaxed the fitting constraints to have only the leading/dominant component (the smallest lifetime $\tau_{1}$ ) shared across the dataset, including the test dose signals. Figure 4 shows a representative summary of the results from fitting three of the six samples used to measure extended dose responses. At first glance, all the fits in Fig. 
4a-c appear to pass through the data points, and the residuals for the three natural signals (shown below) have no structure. However, the residuals from the regenerated curves show significant oscillations about 0 during the initial $50 \mathrm{~s}$ of the stimulation, progressively increasing at higher doses. Had the same three exponential components (with different intensities) been present both in the natural and regenerated curves, the residuals would have lacked any oscillatory behaviour, and the misfit would not become worse with increasing dose.

Oscillatory trends in the residuals of the regenerated signals can be explained in two ways: (i) the assumed multi-exponential model is inadequate and failing to describe the VSL decay as a simple sum of three first-order systems, or (ii) three components are not enough to describe the data. Although the natural decays show no apparent trends or oscillations in the residuals, their signal to noise ratio is too low to validate the component breakdown in question (cf. Istratov and Vyvenko, 1999); for the much brighter regenerated signals, three components clearly appear as too few to give a plausible fit. Since adding more components has negligible effect on the explained variance, we deem multi-exponential analysis with more than three components as prone to overfitting, and hence proceed to regard the VSL decay curves from the Luochuan section as non-exponential in character and at least partly governed by non-first order kinetics as suggested by Ankjærgaard et al. (2013). The extraction of shared component(s) from the natural and regenerated signals demands an extensive study on its own and goes beyond the scope of this paper. We therefore proceed with two simple integrations of the VSL decay curve and then evaluate whether this is a satisfactory approach in obtaining reliable equivalent dose values from the SAR dose response curves.

\subsection{Dose-response curves and equivalent-dose estimates}

A comparison between the natural $\left(\mathrm{L}_{n} / \mathrm{T}_{\mathrm{n}}\right)$ and the regenerated $\left(\mathrm{L}_{\mathrm{x}} / \mathrm{T}_{\mathrm{x}}\right)$ dose response curves of the VSL signal is shown in Fig. 5a, and was obtained by integrating both types of decay curves using the same late background integration (initial $2.5 \mathrm{~s}$ minus the last $25 \mathrm{~s}$ ). The SAR DRCs are all tightly clustered (data not shown) suggesting the existence of a standardized laboratory dose response curve; hence, for each $\mathrm{L}_{\mathrm{x}} / \mathrm{T}_{\mathrm{x}}$ dose point, outliers were removed in a similar fashion to the natural DRC (see Section 3), producing average $\mathrm{L}_{\mathrm{x}} / \mathrm{T}_{\mathrm{x}}$ values plotted in Fig. 5a. The two sets of data (natural and SAR DRCs) seem to be almost parallel (Fig. 5a), but are not overlapping in any part of the dose range. The average SAR DRC can be adequately fitted (adjusted $R^{2}=0.99$; fit not shown) by a sum of two saturating exponentials, yielding characteristic doses of $\mathrm{D}_{0,1} \sim 30 \mathrm{~Gy}$ and $\mathrm{D}_{0,2} \sim 2600 \mathrm{~Gy}$; yet, since it is 
not clear what shape governs the natural DRC, we choose to make no assumption on the functional form of either the natural or regenerated DRCs. Instead, we obtained equivalent doses $\mathrm{D}_{\mathrm{e}}$ for the 24 samples by projecting the natural signals onto a piecewise linear interpolation of the SAR DRC data points (black line shown in Fig. 5a). The resulting $\mathrm{D}_{\mathrm{e}}$ values are shown in Fig. 5b, plotted against their expected natural doses. As anticipated from the non-overlapping DRCs (Fig. 5a), such obtained $\mathrm{D}_{\mathrm{e}}$ values largely underestimate their predictions (by $\sim 70 \%$ on average), with no samples at all falling within $10 \%$ of their expected natural dose.

Attributing the discordance between the natural and SAR DRCs on the late background signal integration limits which intensifies the effect of the re-trapping (Ankjærgaard et al., 2013), we attempted to minimize the contribution from re-trapping and isolate the initial part of the decay curves through early background subtraction (cf. Cunningham and Wallinga, 2010). Here we choose an early background integration similar (but not identical) to that used in Ankjærgaard et al. (2013) as their 'Component A'. Fig. 5c compares the natural and regenerated DRCs for the early background integration (initial $2.5 \mathrm{~s}$ minus the following $15 \mathrm{~s}$ ), and although the two curves are still not overlapping, the gap between the two DRCs is visibly narrower than in Fig. 5a. The early-background SAR DRC can still be adequately fitted with a sum of two saturating exponentials (adjusted $R^{2}=0.99$, fit not shown), yielding $\mathrm{D}_{0}$ values within error of that obtained for the late background subtraction (Fig. 5a), suggesting that varying the integration limits exerts only a minor effect over the SAR DRC shape. Natural signals were converted, as before, into $\mathrm{D}_{\mathrm{e}}$ values by projection onto a piecewise linear interpolation through the SAR DRC, shown in Fig. 5d. Despite that the $D_{e}$ values still largely underestimate their predicted values (by $\sim 50 \%$ on average), early background subtraction brings six of the 24 samples within $10 \%$ of their predicted values, offering a modest improvement over the late background subtraction strategy.

Despite the overall poor agreement of the $\mathrm{D}_{\mathrm{e}}$ values in Fig. $5 \mathrm{~d}$ with independent dose constraints, it is notable that (i) three out of four samples in the <250 Gy range (148101; 148104-05) agree within $10 \%$ with their independently estimated dose, akin to the chronological agreement between BLSL and VSL ages in the 44-224 Gy range (Ankjærgaard et al., 2013), and (ii) there is disagreement of 17 samples in the $>250$ Gy range, akin to the 50 - 80\% underestimates of independently known doses in the 1.6-3 kGy range (Ankjærgaard et al., 2015). This suggests that the age underestimation is caused by a progressive deviation between the natural and laboratory DRCs (e.g. Chapot et al., 2012), rendering SAR DRCs a 
problematic proxy to estimate high equivalent doses, and paving the way to multiple-aliquot schemes described in the second half of this paper.

\subsection{Heating induced trapping sensitivity change}

The main challenge in obtaining VSL $\mathrm{D}_{\mathrm{e}}$ values in agreement with independent estimates lies in the fact that the natural and laboratory generated SAR DRCs are not identical in shape. It has previously been illustrated by Jain (2009) that the shape of the SAR DRC is highly dependent on the preheat temperature, with lower temperatures resulting in lower characteristic doses $\left(\mathrm{D}_{0}\right)$, presumably because of the contribution of other, thermally unstable and early saturating, traps to the main VSL signal. Choosing a preheat of $340{ }^{\circ} \mathrm{C}$ for $10 \mathrm{~s}$, Jain (2009) obtained an acceptable dose recovery at $300 \mathrm{~Gy}$. It was later experimentally demonstrated through a series of dose recovery experiments, that preheating to $\geq 320{ }^{\circ} \mathrm{C}$ (for 1-10 s) yielded the best results (see supplementary table in Ankjærgaard et al., 2013).

Subsequently, Ankjærgaard et al. (2015) lowered this temperature to $300{ }^{\circ} \mathrm{C}$ but increased the duration to $100 \mathrm{~s}$. This combination was chosen to achieve more uniform levels of preheating (given potential fluctuations in thermal contact between heater plate and sample during very short preheats), and yielded acceptable dose recoveries for three out of the four samples tested. Nonetheless, high preheats can be problematic (cf. Wallinga et al. 2000; Kars et al., 2014) because they may increase the charge trapping probability during subsequent irradiation. A high preheat, leading to a significant change in the trapping probability, could imply that the accumulation of the $\mathrm{L}_{\mathrm{n}}$ signal in nature is less efficient than the accumulation of all the subsequent $T_{n}, L_{x}$ and $T_{x}$ signals in the laboratory. The consequence of such behaviour would be that interpolation of $\mathrm{L}_{\mathrm{n}} / \mathrm{T}_{\mathrm{n}}$ onto a series of $\mathrm{L}_{\mathrm{x}} / \mathrm{T}_{\mathrm{x}}$ curves contains an internal bias towards younger age (smaller dose), thus making the interpolation rather unjustified.

To test whether the trapping probability is changed due to the $100 \mathrm{~s}, 300{ }^{\circ} \mathrm{C}$ preheat used prior to measuring the natural and test dose VSL signals, we used a multiple-aliquot additive-dose (MAAD; Aitken, 1998) protocol: 48 aliquots of the youngest sample 148101, each containing a natural dose of $\mathrm{N}=44 \pm 5 \mathrm{~Gy}$, were split into eight batches ( 6 aliquots each), and exposed to differential additive doses, yielding six aliquots with $\mathrm{N}+0, \mathrm{~N}+115, \mathrm{~N}+230$, $\mathrm{N}+460, \mathrm{~N}+920, \mathrm{~N}+1840, \mathrm{~N}+3690$, and N+8100 Gy each. The resulting aliquots were measured using the protocol in Table 2 and each batch was subsequently data-reduced as described in Section 3. The resulting MAAD DRC (closed triangles) is shown in Figure 6 ( $\mathrm{L}_{\mathrm{a}} / \mathrm{T}_{\mathrm{a}}$, with ' $\mathrm{a}$ ' for additive), alongside former results of the natural $\left(\mathrm{L}_{\mathrm{n}} / \mathrm{T}_{\mathrm{n}}\right.$; closed circles) and the SAR DRCs ( $\mathrm{L}_{\mathrm{x}} / \mathrm{T}_{\mathrm{x}}$; open circles); the latter two replotted from Fig. 5c. Excluding the 
highest dose point ( $8144 \mathrm{~Gy}$ ), the MAAD DRC follows the shape of the natural DRC, and is distinctly different in shape from the SAR DRC. This means that irradiation in itself (adding a dose on top of the natural signal) does not noticeably change the trapping sensitivity of the sample.

To investigate whether charge-trapping sensitivity (Wallinga et al., 2000) could be caused by the $300{ }^{\circ} \mathrm{C}$ preheat temperature (step 2 in the VSL protocol, Table 2), the MAAD protocol was repeated for a fresh batch of 48 aliquots, but now with an additional "step 0" of heating to $300{ }^{\circ} \mathrm{C}$ for $100 \mathrm{~s}$ prior to each laboratory added dose. The MAAD DRC with 300 ${ }^{\circ} \mathrm{C}$ heating prior to the added dose irradiation follows the shape of the SAR DRC almost identically (open triangles, Fig. 6). The mismatch between the shapes of $\mathrm{L}_{\mathrm{a}} / \mathrm{T}_{\mathrm{a}}$ with and without the $300{ }^{\circ} \mathrm{C}$ preheat in "step 0 " suggests that this preheat exerts a key influence on the trapping sensitivity of the sample, leaving only the $L_{n}$ or unheated $L_{a}$ signals unaffected. With this knowledge, we can infer that in the natural signals $\mathrm{L}_{\mathrm{n}} / \mathrm{T}_{\mathrm{n}}$, the $300{ }^{\circ} \mathrm{C}$ preheat in step 2 changes the trapping sensitivity of $T_{n}$ and all subsequently measured signals $\left(\mathrm{L}_{x} / \mathrm{T}_{\mathrm{x}}\right)$ of the SAR protocol. Therefore, when $\mathrm{T}_{\mathrm{n}}$ is used to correct the $\mathrm{L}_{\mathrm{n}}$ signal (unaffected by trapping sensitivity change), such obtained $L_{n} / T_{n}$ are all biased below $L_{x} / T_{x}$ ratios, as both $L_{x}$ and $T_{x}$ have experienced an increase in trapping sensitivity during the SAR protocol.

To further determine at what preheat temperature does the charge-trapping sensitivity change becomes significant, twelve batches of three aliquots of sample 148101 were prepared, heated to between 20 and $500{ }^{\circ} \mathrm{C}$ for $100 \mathrm{~s}$, irradiated with $920 \mathrm{~Gy}$, and measured using the first cycle in Table 2, to obtain the $\mathrm{L}_{\mathrm{a}}$ and $\mathrm{T}_{\mathrm{a}}$ signals. Note that in contrast to the experiments of Wallinga et al. (2000) and Kars et al. (2014), the natural dose was negligible $(<5 \%)$ in relation to the added dose, and hence was not bleached prior to irradiation. The samples were then all annealed to $500{ }^{\circ} \mathrm{C}$ for $20 \mathrm{~s}$, and given a second test dose to correct the $\mathrm{L}_{\mathrm{a}}$ for potential differences in luminescence brightness between aliquots (i.e. a correction for the potential difference in the number of grains (mass) on the aliquots given by $L_{a} / T_{x 2}$ ). Figure 7 shows the relationship between VSL trapping sensitivity and pre-irradiation temperature where each point is the average over three mass-corrected aliquots. This dataset suggests that the change in sensitivity stays fairly constant up until about $300{ }^{\circ} \mathrm{C}$, after which it increases rapidly. From the inset to Fig. 7, it is also evident that it is not a change in trapping sensitivity that causes the differences in VSL decay curve shape between the natural and the regenerated signals (Fig. 3), as the VSL decay curves in Fig. 7 are identical. The dose dependent slowness of the regenerated VSL decays of Fig. 3 is more likely to originate from re-trapping during the stimulation as discussed earlier in Section 4.1. 
Although the $300{ }^{\circ} \mathrm{C}$ preheat prior to irradiation and VSL measurements seems to be on the edge of inducing a significant trapping sensitivity change (Fig. 7), this effect could contribute to the $D_{e}$ underestimations observed in Fig. 5. To this end, we re-measured the natural and SAR DRCs using a preheat of $260{ }^{\circ} \mathrm{C}$ and a test dose preheat of $240{ }^{\circ} \mathrm{C}$ (both for $100 \mathrm{~s}$ ); these chosen temperatures are both in the region of no significant trapping sensitivity change in Fig. 7. The natural $\mathrm{L}_{\mathrm{n}} / \mathrm{T}_{\mathrm{n}}$ measurements were carried out on a subset of the 24 samples, focusing on the 16 samples with expected $D_{e}$ values between $~ 340$ and $1700 \mathrm{~Gy}$ (samples 148106-148123), and the SAR DRCs were measured on an aliquot each from samples 148110 and 148114 . The natural and average SAR DRC curves for this alternative preheating protocol are shown in Fig. 8a. Unfortunately, the result of lowering the temperature of the heating steps does not improve the similarity between the shapes of the natural and SAR DRCs; on the contrary, the natural DRC appears to be slightly lower relative to the SAR DRC compared to that in Fig. 5c (with high preheating regime). This is further evident when the interpolated $D_{e}$ values are plotted against their expected doses in Fig. 8b, with only two samples agreeing with their expected doses. While this study did not exhaust all the possibilities of optimising the SAR protocol to eventually yield meaningful agreement between expected and deduced ages, further attempts in this direction remain beyond the scope of this paper.

\section{Multiple-aliquot additive-dose (MAAD) response}

\subsection{Dose-response curves and equivalent-dose estimates}

Although the conventional method of estimating palaeodoses using a SAR protocol has proven difficult for the VSL signal, the multiple-aliquot additive-dose approach shown in Fig. 6 appears much more encouraging as the natural and MAAD DRCs are very similar in shape. Figure 9 compares the natural and natural+added dose decay curves (using the same four samples as the natural vs. SAR decay curve shape comparison, Fig. 3), and it is clear that the MAAD decay curves succeed in reproducing the shapes of the natural signals, especially in the initial part of the decay (Fig. 9). Note that the four natural decay curves do get slightly slower with increase in dose, but additive doses on top of the youngest natural sample (Fig. 9a) were able to mimic the natural slowing-down (Fig. 9b-d), compared to the SAR protocol where the shape change was much more pronounced (Fig. 3a-d). Because the natural and MAAD decay shapes are similar during the initial part of the curves, an early background subtraction is likely to isolate a similar part across the signals and result in similar natural and MAAD DRCs. 
Fig. 10a demonstrates that if we exclude the highest-dose point ( $8144 \mathrm{~Gy}$, justified by its being far beyond the estimated $2 \mathrm{D}_{0}$ saturation limit of $\mathrm{L}_{\mathrm{n}} / \mathrm{T}_{\mathrm{n}}$; see Fig. 2), the MAAD DRC neatly overlaps with the natural DRC (replotted from Fig. 6), indicating that no trapping sensitivity change has been induced by acquisition of the additive dose. Making no assumptions about the functional form of the DRCs, we can project 22 out of the 24 natural signals onto the MAAD DRC using piecewise linear interpolation between its dose points as before. The resulting $D_{e}$ values are shown in Fig. 10b; note that the two youngest samples have been omitted as sample 148101 was used to construct the MAAD DRC (and hence cannot be interpolated onto itself), while sample 148102 had an anomalously low $L_{n} / T_{n}$ intensity which does not interpolate onto the MAAD DRC at all. The remaining $22 \mathrm{VSL} \mathrm{D}_{\mathrm{e}}$ values appear evenly distributed around the 1:1 line suggesting broad agreement with the expected natural doses up until about $\sim 1800 \mathrm{~Gy}$, beyond which the $\mathrm{D}_{\mathrm{e}}$ values scatter significantly.

\subsection{MAAD age estimations for the Luochuan section}

Using the laboratory measured environmental dose rates for each of the 22 samples listed in Table 1, the equivalent doses (Fig. 10b) are converted into VSL ages and plotted in Fig. 11 against the existing independent age control for the Luochuan section. Approximating the growth of the natural VSL DRC via a saturating exponential plus an offset as discussed in Section 3 and Fig. 2, we can adopt the classical 2D $\mathrm{D}_{0}$ criteria (Wintle and Murray, 2006) as a conservative upper limit for meaningful interpolation of the natural luminescence intensities onto the MAAD DRC. With 2D $\mathrm{D}_{0}=1842 \pm 444 \mathrm{~Gy}$, and a site-averaged environmental dose rate of $3.10 \pm 0.05 \mathrm{~Gy} / \mathrm{ka}$ for the 22 samples, the shaded grey zone in Fig. 11 beyond $\sim 600 \mathrm{ka}$ $(594 \pm 143 \mathrm{ka})$ corresponds to the area where the natural signals cannot be distinguished from full saturation with a high degree of certainty. Out of the 22 VSL ages, 15 are in agreement with their independent age controls within their 'one standard error' joint uncertainties (shown with black symbols, see also Table 1). Of the remaining 7 samples which do not yield expected ages (open grey symbols), three show a significant age overestimation, and four fall beyond the trustable dating limit discussed in Section 5.1. Of the samples in the datable range of $<600 \mathrm{ka}$, we obtain VSL ages in agreement with independent age control for more than $80 \%$ of the samples, which is a major improvement over the $D_{e}$ results using the SAR VSL protocol (Fig. 5d), where the success ratio was much lower (25\%).

We have demonstrated that VSL can obtain ages in agreement with independent age control up until $\sim 600 \mathrm{ka}$ at the Luochuan type section using a VSL MAAD protocol. This 
appears as a noteworthy extension of the datable age range at this site, when compared to the upper limits of quartz BLSL ( 50 ka; Buylaert et al., 2007; Lai, 2010; Chapot et al., 2012), feldspar MET post-IR IRSL ( 450 ka; Li et al., 2014), and marginally even the lifetimecorrected TT-OSL ( 500 ka; Chapot et al., in press). While largely similar to the $\sim 0.5 \mathrm{Ma}$ age limit of TT-OSL, VSL has the advantage of being free of the uncertainties propagating into a TT-OSL lifetime correction, namely (i) the Arrhenius parameters of the target trap, and (ii) the burial temperature(s) of the sediment throughout its geological history. The thermal and athermal stability of VSL (Ankjærgaard et al., 2013; 2015) makes it a direct measurable quantity, which requires neither an age adjustment for signal loss (increasingly imprecise at old ages), nor the justification of its absence (not always straightforward in feldspar luminescence dating). While further modifications and improvements will be needed, it appears that a MAAD-type protocol is a promising step forward in our attempts to extend the luminescence dating range, using quartz VSL in particular.

\section{Discussion}

\subsection{Independent age control}

The expected natural doses, which form the basis of this and other luminescence dating studies at Luochuan, are all anchored in an inherently relative chronology (CHILOPARTS, Chinese Loess Particle Timescale), in which sediment properties are matched to obliquity and precession cyclicity in the Earth's spin. Although in line with other relative chronologies at this site, including marine isotopic stages and palaeomagnetic reversal data (Ding et al., 2002), there rises a certain (typically unmentioned) conceptual problem in testing or validating an absolute dating method (such as VSL) against a relative chronology. While our primary trend (i.e. VSL intensity increase with geological age) is compelling and solid, it is hard to evaluate how much of the scatter is due to natural variability in the VSL signals, and how much arises from imprecisions of the relative chronology. While our assigned $10 \%$ relative errors on CHILOPARTS are quite generous and realistic, it is important to highlight the future necessity to test VSL (and other age-extending protocols) in sediments securely dated by similarly absolute, and hopefully multiple, independent chronometers.

\subsection{Natural DRC}

We used 24 samples from the Luochuan section, spanning across two and a half orders of magnitude in expected natural doses (40 - $8000 \mathrm{~Gy}$ ), to construct a natural VSL dose 
response curve. The aim of this work was to investigate whether and how laboratory regenerated dose response curves can reproduce the natural signal growth, and thereby provide reliable VSL ages. Due to large inter-sample scatter and only a few samples in the low ( $<200 \mathrm{~Gy}$ ) dose range, it is difficult to quantify the shape of the natural dose response curve, although it can be concluded that it shows significant growth as a function of natural expected dose until 1800 Gy (Fig. 2). Here we chose to fit the natural DRC with an exponential function (with a constant offset), equivalent to the approach of Jain (2009), in which all regenerative DRCs show a clear offset at zero dose, similar to the natural DRC presented here. It is not unlikely that the natural DRC has an offset at the origin, as VSL doses measured in modern samples are reported to range between 10 and 35 Gy (Ankjærgaard et al., 2013; Hernandez and Mercier, 2015), indicating that there exists a hard-to-bleach component in the natural VSL signal preventing the growth curve to pass through the origin at $0 \mathrm{~Gy}$. The existence, and magnitude of this hard-to-bleach dose offset needs to be investigated further by additional measurements of samples with natural doses $<200 \mathrm{~Gy}$, but is expected to be only of marginal importance for the dose range of interest targeted by the VSL signal.

\subsection{Natural vs. regenerated DRCs}

Comparison of the natural DRC $\left(\mathrm{L}_{\mathrm{n}} / \mathrm{T}_{\mathrm{n}}\right)$ and the SAR DRC $\left(\mathrm{L}_{\mathrm{x}} / \mathrm{T}_{\mathrm{x}}\right)$ growth (Fig. 5a,c) using two different signal integration ranges reveals two main differences: (i) the natural and SAR DRCs are markedly dissimilar in shape, and overlap only for the youngest sample, and (ii) the growth of the SAR DRC continues beyond $1800 \mathrm{~Gy}$, while the natural DRC becomes saturated. A possible origin of the mismatch between the DRCs (Fig. 3) is that the VSL decay curves from the SAR protocol cannot regenerate the shape of the natural VSL decay curves. Confirmed by component fitting, the notion of dissimilarity between natural and regenerated SAR decay curves undermines the straightforward application of a SAR-type protocol for VSL; Fig. 4 illustrates that no simple first-order model can simultaneously describe both the natural and the regenerated signals, and that the decay shapes are most likely governed by non-first order kinetics such as re-trapping (Ankjærgaard et al., 2013).

Through reintroducing the MAAD approach (Aitken, 1998) and comparing two MAAD DRCs, one with and another without a heating step prior to sample irradiation (open and filled triangles in Fig. 6, respectively), it becomes evident that the $300{ }^{\circ} \mathrm{C}$ preheat (also used in the SAR protocol; Table 2) is likely introducing a change in the trapping efficiency between the natural and all the subsequently measured signals. This change in trapping efficiency could in part be responsible for the equivalent dose underestimations seen in Fig. 5. 
However, adopting a lower preheat $\left(260\right.$ and $\left.240{ }^{\circ} \mathrm{C}\right)$ results in an even larger dose underestimation, due to the contribution from shallower and less stable traps (Jain, 2009) or problems with dose recovery. Preheating of VSL signals thus appears to be a double-sided sword: a low preheat does not isolate a sufficiently stable VSL signal, whereas a high preheat is possibly affected by a trapping sensitivity change. Even though there might exist an optimum preheat temperature that balances between these two phenomena, we have not managed to find it in the present study. Fearing that such an optimum might further be sample-dependent, we at present consider the SAR VSL protocol as quite problematic for a correct estimation of VSL doses and ages.

The VSL signal discussed above in the different experiments is used as a proxy to investigate how charge is trapped, and migrate within a crystal. However, the relationship between dose and observed luminescence is more complex than generally assumed because the observed luminescence signal only corresponds to a fraction of the originally trapped charge. An alternative approach to examine trapped charge directly - and thereby the effect of preheating on the trapping efficiency - is via the measurement of exo-electron emission. During optical (or thermal) stimulation, a fraction of the trapped electrons receive enough energy to escape the crystal through the conduction band. The advantage of measuring the exo-electrons is therefore, that the signal is derived directly from trapped charge, and so the relationship to the trapped population is less complex than in the case of luminescence (Ankjærgaard et al., 2006; 2008). Recording and comparing exo-electron and luminescence signals would allow insight into the relative importance of electron traps and recombination centres during the SAR VSL protocol measurements, and could possibly identify how preheating affects the natural and the regenerated VSL signals differently.

Deeming the high preheat as one of the central problems of the SAR VSL dose underestimations at Luochuan (Fig. 6), it is instructive to examine a geological scenario, in which a high preheat prior to irradiation has occurred in nature (thus mimicking a high preheat in the laboratory). Such is the case with palaeosols that have been naturally baked by overlying basalts, and thus subjected to a natural high-temperature heating prior to their subsequent irradiation. If the effect of heating the sedimentary quartz grains in nature is equally irreversible as heating in the laboratory, no further change should be observed in the trapping efficiency, thereby making the SAR protocol applicable. Such a site was studied in Ankjærgaard et al. (2015), where quartz from three palaeosols, capped by basalt flows (K-Ar ages spanning from 1.58 to $0.96 \mathrm{Ma}$ ), have experienced a thermal resetting of $>300{ }^{\circ} \mathrm{C}$ at the time of basalt emplacement. Although such a natural heating event should make natural and 
laboratory irradiations comparable, all three samples still underestimated their corresponding K-Ar ages by $\sim 50 \%$ or more. Given these results, we suggest that application of SAR methods for VSL dating is highly problematic, due to VSL properties with regards to (i) the trapping sensitivity change induced by preheating, and (ii) the decay shape change. For this reason, we changed our focus to different methods for VSL dose estimation.

\subsection{Implications for dating}

The advantage of the MAAD protocol over SAR, is that it preserves the overall VSL decay shape between the natural and the additive-dose signals (Fig. 9), and does not demonstrably affect the trapping sensitivity of the signal (Fig. 10a). Although the luminescence dating community has, in the past decades, shifted from additive-dose approaches to regenerative dose methods, both are fundamental methodologies (Aitken, 1998) and this study demonstrates a clear advantage in re-exploring the MAAD technique in the context of the VSL signal. Although the MAAD DRC presented here $(n=6$ per dose point; data reduced) has a noticeably larger scatter than the SAR protocol, we have demonstrated that this scatter can be considerably reduced in statistically-powerful sample sizes (here shown for $n=54)$.

In a standard MAAD protocol, a series of increasing doses are added to the natural sample, and the resultant dose response is extrapolated back to negligible light level to obtain the $\mathrm{D}_{\mathrm{e}}$. A remaining challenge of applying the MAAD protocol in VSL dating, is the current large uncertainty regarding the true shape of the natural VSL dose response curve at low doses (Fig. 2), causing such an extrapolation to be unreliable at present. To circumvent this problem, there are two possible approaches: (i) a modern analogue, or (ii) an un-invasive bleaching of the natural VSL signal. For the former approach, a zero-age, or a comparably young and well-dated natural analogue is needed, to form the base for the additive dose response curve onto which the natural signal is to be interpolated. For the Luochuan site, where sedimentation has been almost constant throughout the Quaternary, with a persistent sedimentary provenance, this requirement is easily addressable by taking representative sediments from the top of the loess section. However, given the considerable reworking of the Earth's surface during the Quaternary, finding modern analogues for million-year old deposits might not only be geologically challenging, but also perhaps technically impossible. The second possibility, circumventing the necessity to find a modern analogue, is to use a multiple aliquot regenerative (MAR) dose type protocol (Aitken, 1998) or the Australian slide method, the latter combining MAAD and MAR (Prescott et al., 1993), where the natural VSL signal is 
"reset" in a non-invasive manner prior to its laboratory irradiation and age estimation. Further exploration of the abovementioned possibilities is currently underway.

\section{Conclusions}

The natural dose response curve of VSL at Luochuan shows a clear correlation $(\mathrm{r}=$ 0.88 ) between $\mathrm{L}_{\mathrm{n}} / \mathrm{T}_{\mathrm{n}}$ and $\log _{10}($ dose $)$, demonstrating that the quartz VSL signal has potential for dosimetry in the $200-2000$ Gy range, and is of interest for dating beyond typical age limitations of other luminescence methods. Despite the inconclusiveness regarding the functional form of the natural dose response curve, its natural signal exhibits steady growth up to $\sim 1800 \mathrm{~Gy}$. This implies that VSL would enable dating at Luochuan up to $\sim 600 \mathrm{ka}$, and possibly further back in time in other environments associated with lower dose rate.

Comparison of the natural and SAR dose response curves reveals that they do not overlap, causing large underestimations in equivalent doses. This likely originates in two main problems in the SAR protocol, namely: (i) a significant difference in the decay curve shape between the natural and the regenerative doses, and (ii) a trapping sensitivity change between the natural and all subsequent regenerated signals due to the preheat used in the protocol. The decay curve shape difference cannot be circumvented by multi-exponential component fitting, and appears to be an inherent feature, likely to be related to charge retrapping. Furthermore, the trapping sensitivity change undermines the interpolation of the natural signal onto the regenerated growth curve, at least partly causing the observed underestimations in equivalent dose.

To overcome the issues related to the SAR protocol, we resort to a MAAD approach, in which differential doses are accumulated on top of multiple aliquots of the youngest available geological sample. This approach circumvents the trapping sensitivity change problem and produces comparable natural and added-dose decay curves. The MAAD protocol yields VSL ages in agreement with the CHILOPARTS chronology up until $~ 600$ ka for 15 samples, beyond which the natural signal becomes indistinguishable from saturation and should not be used for dating. Application of the MAAD protocol appears as a promising approach in VSL dating and will be tested in other sites with absolute independent chronology.

\section{Acknowledgements}

This project has been financed by the Netherlands Organisation for Scientific Research, NWO VENI (grant 863.13.023) to C. Ankjærgaard, and B. Guralnik acknowledges NWO VENI 
(grant 863.15.026). J.-P. Buylaert thanks the Danish Council for Independent Research Natural Sciences (FNU) for financial support (Steno grant no. 11-104566). We thank Melissa Chapot and an anonymous reviewer for a critical reading of the manuscript providing helpful and constructive comments.

\section{References}

Adamiec, G., Duller, G.A.T., Roberts, H.M., Wintle, A.G., 2010. Improving the TT-OSL SAR protocol through source trap characterisation. Radiation Measurements 45, 768-777. Aitken, M.J., 1998. An introduction to optical dating: the dating of Quaternary sediments by the use of photon-stimulated luminescence. Oxford University Press.

Ankjærgaard, C., Denby, P.M., Murray, A.S. and Jain, M., 2008. Charge movement in grains of quartz studied using exo-electron emission. Radiation Measurements 43, 273-277.

Ankjærgaard, C., Guralnik, B., Porat, N., Heimann, A., Jain, M., Wallinga, J., 2015. Violet stimulated luminescence: geo-or thermochronometer? Radiation Measurements 81, 78-84.

Ankjærgaard, C., Jain, M., Wallinga, J., 2013. Towards dating quaternary sediments using the quartz violet stimulated luminescence (VSL) signal. Quaternary Geochronology 18, 99109.

Ankjærgaard, C., Murray, A.S., Denby, P.M. and Bøtter-Jensen, L., 2006. Measurement of optically and thermally stimulated electron emission from natural minerals. Radiation Measurements 41, 780-786.

Buylaert, J.P., Jain, M., Murray, A.S., Thomsen, K.J., Thiel, C., Sohbati, R., 2012. A robust feldspar luminescence dating method for Middle and Late Pleistocene sediments. Boreas $41,435-451$.

Buylaert, J.P., Vandenberghe, D., Murray, A.S., Huot, S., De Corte, F., Van den haute, P., 2007. Luminescence dating of old (>70 ka) Chinese loess: a comparison of single-aliquot OSL and IRSL techniques. Quaternary Geochronology 5, 9-14.

Chambers, J.M., Cleveland, W.S., Kleiner, B. and Tukey, P.A., 1983. Graphical Methods for Data Analysis. Wadsworth \& Brooks/Cole.

Chapot, M.S., Roberts, H.M., Duller, G.A.T., Lai, Z.P., 2012. A comparison of natural- and laboratory-generated dose response curves for quartz optically stimulated luminescence signals from Chinese Loess. Radiation Measurements 47, 1045-1052. 
Chapot, M.S., Duller, G.A.T., Roberts, H.M., 2014. Assessing the impact of pulsedirradiation procedures on the thermally transferred OSL signal in quartz. Radiation Measurements 65, 1-7.

Chapot, M.S., Roberts, H.M., Duller, G.A.T., Lai, Z.P., accepted. Natural and laboratory TTOSL dose response curves: testing the lifetime of the TTOSL signal in nature. Radiation Measurements, DOI: 10.1016/j.radmeas.2015.11.008.

Cunningham, A.C., Wallinga, J., 2010. Selection of integration time intervals for quartz OSL decay curves. Quaternary Geochronology 5, 657-666.

Ding, Z.L., Derbyshire, E., Yang, S.L., Yu, Z.W., Xiong, S.F., Liu, T.S., 2002. Stacked 2.6Ma grain size record from the Chinese loess based on five sections and correlation with the deep-sea $\delta^{18} \mathrm{O}$ record. Paleoceanography $17,1033$.

Duller, G.A.T., Wintle, A.G., 2012. A review of the thermally transferred optically stimulated luminescence signal from quartz for dating sediments. Quaternary Geochronology 7, 6-20.

Guérin, G., Mercier, N., Adamiec, C., 2011. Dose-rate conversion factors: update. Ancient TL $29,5-8$.

Guralnik, B., Ankjærgaard, C., Jain, M., Murray, A.S., Müller, A., Wälle, M., Lowick, S.E., Preusser, F., Rhodes, E.J., Wu, T.S., Mathew, G., 2015. OSL-thermochronometry using bedrock quartz: A note of caution. Quaternary Geochronology 25, 37-48.

Hernandez, M., Mercier, N., 2015. Characteristics of the post-blue VSL signal from sedimentary quartz. Radiation Measurements 78, 1-8.

Jain, M., 2009. Extending the dose range: probing deep traps in quartz with $3.06 \mathrm{eV}$ photons. Radiation Measurements 44, 445-452.

Jain, M., Bøtter-Jensen, L., Murray, A.S., Denby, P.M., Tsukamoto, S., Gibling, M.R., 2005. Revisiting TL: dose measurement beyond the OSL range using SAR. Ancient TL 23, 9-24. Joordens, J.C., d'Errico, F., Wesselingh, F.P., Munro, S., De Vos, J., Wallinga, J., Ankjærgaard, C., Reimann, T., Wijbrans, J.R., Kuiper, K.F. and Mücher, H.J. et al., 2015. Homo erectus at Trinil on Java used shells for tool production and engraving. Nature 518, $228-231$

Kars, R.H., Busschers, F.S., Wallinga, J., 2012. Validating post IR-IRSL dating on Kfeldspars through comparison with quartz OSL ages. Quaternary Geochronology 12, 7486.

Kars, R.H., Reimann, T., Wallinga, J., 2014. Are feldspar SAR protocols appropriate for postIR IRSL dating? Quaternary Geochronology 22, 126-136. 
Lai Z.P., 2010. Chronology and the upper dating limit for loess samples from Luochuan section in the Chinese Loess Plateau using quartz OSL SAR protocol. Journal of Asian Earth Sciences 37, 176-185.

Li, B., Li, S.-H., 2012. Luminescence dating of Chinese loess beyond 130 ka using the nonfading signal from K-feldspar. Quaternary Geochronology 10, 24-31.

Li, B., Li, S.-H., Wintle, A.G., Zhao, H., 2008. Isochron dating of sediments using luminescence of K-feldspar grains. Journal of Geophysical Research: Earth Surface (20032012), 113(F2).

Li, B., Roberts, R.G., Jacobs, Z., Li, S.-H., 2014. A single-aliquot luminescence dating procedure for K-feldspar based on the dose-dependent MET-pIRIR signal sensitivity. Quaternary Geochronology 20, 51-64.

Liu, T., 1985. Loess and the Environment. China Ocean Press, Beijing, China

Lu, H., Liu, X., Zhang, F., An, Z. and Dodson, J., 1999. Astronomical calibration of loesspaleosol deposits at Luochuan, central Chinese Loess Plateau. Palaeogeography, Palaeoclimatology, Palaeoecology 154, 237-246.

Murray, A.S., Marten, R., Johnston, A., Martin, P., 1987. Analysis for naturally occurring radionuclides at environmental concentrations by gamma spectrometry. Journal of Radioanalytical and Nuclear Chemistry 115, 263-288.

Murray, A.S., Wintle, A.G., 2003. The single aliquot regenerative dose protocol: potential for improvements in reliability. Radiation Measurements 37, 377-381.

Prescott, J.R., Huntley, D.J. and Hutton, J.T., 1993. Estimation of equivalent dose in thermoluminescence dating - the Australian slide method. Ancient TL 11, 1-5.

Prescott, J.R., Hutton, J.T., 1994. Cosmic ray contributions to dose rates for luminescence and ESR dating: large depths and long-term time variations. Radiation measurements 23, 497500 .

Preusser, F., Chithambo, M.L., Götte, T., Martini, M., Ramseyer, K., Sendezera, E.J., Susino, G.J., Wintle, A.G., 2009. Quartz as a natural luminescence dosimeter. Earth-Science Reviews 97, 184-214.

Singarayer, J.S., Bailey, R.M., Rhodes, E.J., 2000. Potential of the slow component of quartz OSL for age determination of sedimentary samples. Radiation Measurements 32, 873-880. Thiel, C., Buylaert, J.-P., Murray, A.S., Elmejdoub, N., Jedoui, Y., 2012. A comparison of TT-OSL and post-IR IRSL dating of coastal deposits on Cap Bon peninsula, north-eastern Tunisia Christine. Quaternary Geochronology 10, 209-217. 
Timar-Gabor, A., Wintle, A.G., 2013. On natural and laboratory generated dose response curves for quartz of different grain sizes from Romanian loess. Quaternary Geochronology $18,34-40$.

Vandenberghe, D., De Corte, F., Buylaert, J.P., Kučera, J., Van den haute, P., 2008. On the internal radioactivity in quartz. Radiation Measurements 43, 771-775.

Wallinga, J., Murray, A., Duller, G., 2000. Underestimation of equivalent dose in singlealiquot optical dating of feldspars caused by preheating. Radiation Measurements 32, 691695.

Wang, X.L., Lu, Y.C., Wintle, A.G., 2006. Recuperated OSL dating of fine-grained quartz in Chinese loess. Quaternary Geochronology 1, 89-100.

Wintle, A.G., 2008. Luminescence dating: where it has been and where it is going. Boreas 37, 471-482.

Wintle, A.G., Murray, A.S., 2006. A review of quartz optically stimulated luminescence characteristics and their relevance in single-aliquot regeneration dating protocols.

Radiation Measurements 41, 369-391.

Istratov, A.A., Vyvenko, O.F., 1999. Exponential analysis in physical phenomena. Review of Scientific Instruments 70, 1233-1257.

\section{Table captions}

Table 1

Summary of samples from the Luochuan site used for this study, listing the sample codes (col. 1), location within stratigraphic unit (col. 2), expected age (col. 3), measured radionuclide activities using high resolution gamma spectrometry (cols. 5-8), assumed life-time average water content $\left({ }^{*}\right.$ C.W. in \% dry weight) (col. 9), total environmental dose rate to $63-90 \mu \mathrm{m}$ quartz (col. 10), expected natural dose (col. 11), measured MAAD VSL dose (col. 12), and measured MAAD VSL age (col. 13). For all samples except 148101-02 the expected age is based on the loess-palaeosol stratigraphy (Ding et al., 2002) with a presumed 10\% error. The expected age and dose of samples 148101-02 are based on direct quartz SAR-BLSL chronology, which is about twice older than their CHILOPARTS age in Ding et al., 2002 (5.0 \pm 0.5 and $12.0 \pm 1.2 \mathrm{ka}$, respectively).

Table 2

VSL protocol used for both SAR and MAAD in this study. 


\section{Figure captions}

Figure 1

Natural VSL decay curves from six samples (148102; -06; -10; -18; -21; -25) spanning across expected natural doses between $\sim 60$ and $\sim 7900$ Gy. Shown are (a) the absolute intensity of a representative aliquot per sample, and (b) the normalised intensity of the samples, from summed 6 aliquots per sample (the normalising factor is the integral of the initial $0.5 \mathrm{~s}$ of summed intensities of the corresponding test dose of $540 \mathrm{~Gy}$ ). Insets: same data plotted on a logarithmic time scale for better visualisation of the initial decay shape.

Figure 2

Natural VSL dose response curve for the Luochuan site using the 24 samples listed in Table 1. For each sample, the mean $\mathrm{L}_{\mathrm{n}} / \mathrm{T}_{\mathrm{n}}$ and standard deviation from six subsamples (see text for details on data reduction) is plotted against the expected natural dose. Signals were analysed using late background subtraction (initial $2.5 \mathrm{~s}$ minus the last $25 \mathrm{~s}$ ). The data is fitted with a single saturating exponential function plus a constant offset (red full line), giving a $\mathrm{D}_{0}$ value of $921 \pm 222$ Gy. The dashed blue lines indicate the $95 \%$ confidence intervals of the fit. Inset: same data shown on a log dose scale.

Figure 3

Summed natural VSL decay shape (circles) compared to those from SAR signal decays (lines). Each natural decay is the sum of six individual decay measurements. All curves have been background subtracted (at $490 \mathrm{~s}$ ) and normalised to the initial point. Note that only the initial $100 \mathrm{~s}$ of the decay curves are shown. The subplots show the comparison of different natural signal decays and their corresponding regenerated dose decays, using samples (a) 148101 with a natural dose of $45 \mathrm{~Gy}$, (b) 148110 with expected natural dose of $630 \mathrm{~Gy}$, (c) 148123 with expected natural dose of $1700 \mathrm{~Gy}$, and (d) 148125 with expected natural dose of 7900 Gy. For legend of regenerative doses, see (d).

Figure 4

Fitting of natural VSL decay (hollow circles) and three regenerated dose decays (filled circles) for samples (a) 148101, (b) 148113, and (c) 148125, using three exponentially decaying functions and a constant, $c: L_{\mathrm{VSL}}(t)=a_{1} \cdot \exp \left(-t / \tau_{1}\right)+a_{2} \cdot \exp \left(-t / \tau_{2}\right)+a_{3} \cdot \exp \left(-t / \tau_{3}\right)+c$, where $a_{i}$ is the intensity of the component with lifetime $\tau_{i}$. The fits are shown as red lines in 
the top row, and the residuals for the four fits are shown in the four rows immediately below. For each sample, the natural and regenerated signals have been measured using the same aliquot.

\section{Figure 5}

(a) Comparison of natural DRC with SAR DRC for late background subtraction (initial $2.5 \mathrm{~s}$ minus the last $25 \mathrm{~s}$ ); the shown SAR DRC has been constructed by data reduction on each $\mathrm{L}_{\mathrm{x}} / \mathrm{T}_{\mathrm{x}}$ dose point using six SAR DRCs (as with the natural signals), resulting in the average $\mathrm{L}_{\mathrm{x}} / \mathrm{T}_{\mathrm{x}}$ values shown. Note that error bars are often smaller than symbols. (b) By using linear interpolation of the natural signals onto the SAR DRC shown in (a), the resulting equivalent doses, $D_{\mathrm{e}}$, are plotted as a function of the expected natural doses. (c) Comparison of natural DRC with SAR DRC for early background subtraction (initial $2.5 \mathrm{~s}$ minus the following $15 \mathrm{~s}$ ), and (d) the resulting equivalent doses from linear interpolation. Both integration intervals causes large underestimations of the VSL $\mathrm{D}_{\mathrm{e}}$ 's compared to the expected doses.

\section{Figure 6}

Comparison of natural, SAR and MAAD DRC curves using early background subtraction. The natural DRC (black filled circles) and average SAR DRC (open black circles) are reproduced here from Fig. 5c. The MAAD DRC (filled red triangles) was measured using sample 148101 where the added doses $(0,115,230,460,920,1840,3690$, and $8100 \mathrm{~Gy})$ were delivered on top of the natural dose $(44 \pm 5 \mathrm{~Gy}$, see Table 1$)$, after which the MAAD $\mathrm{L}_{\mathrm{a}}$ and $\mathrm{T}_{\mathrm{a}}$ signals were measured. Finally, a prior heated MAAD DRC (open blue triangles) was constructed by heating the aliquots from sample 148101 to $300{ }^{\circ} \mathrm{C}$ for $100 \mathrm{~s}$ prior to delivering the added dose and measuring the resulting $L_{a}$ and $T_{a}$ signals. For both of the heated and unheated MAAD dose response curves, six aliquots were used per dose point and data reduced as described in Section 3.

\section{Figure 7}

Change in VSL trapping efficiency as a function of heating prior to irradiation. Twelve batches of three fresh aliquots of sample 148101 were heated to $20,100,125,150,175,200$, $225,250,275,300,400$, and $500{ }^{\circ} \mathrm{C}$ for $100 \mathrm{~s}$. Following heating, the aliquots were measured using step 1-9 in Table 2 using a dose of 920 Gy (step 1). The aliquots were then given a second test dose (540 Gy; step 5) and measured using steps 6-9. The average VSL signals (step 4) is then 'mass' normalised (see main text) with the VSL signal from the second test 
dose $\left(\mathrm{L}_{\mathrm{a}} / \mathrm{T}_{\mathrm{x} 2}\right)$ and plotted as a function of initial temperature, using an early background subtraction. Inset: VSL decay curve comparison for different initial heating temperatures, the curves have been background subtracted and normalised to the initial point.

\section{Figure 8}

Natural and regenerated DRCs and $\mathrm{D}_{\mathrm{e}}$ measured using reduced temperatures during the heating steps, namely $260{ }^{\circ} \mathrm{C}$ in step 2 , and $240{ }^{\circ} \mathrm{C}$ in step 6 in the VSL protocol from Table 2. (a) Comparison of natural DRC (using the 16 samples 148106-148123) with the average SAR DRC (using samples 148110 and 148114) for early background subtraction, see main text for further detail. (b) Using linear interpolation of the natural signals onto the SAR DRC shown in (a), the resulting equivalent doses, $D_{e}$, are plotted as a function of the expected natural doses.

\section{Figure 9}

Average natural VSL decay shape (circles) comparison to those from MAAD signal decays (lines) using sample 148101. All natural and MAAD decays are the sum of six individual decay measurements. See caption of Fig. 6 for details on the MAAD DRC. All curves have been background subtracted (at $490 \mathrm{~s}$ ) and normalised to the initial point. Note that only the initial $100 \mathrm{~s}$ of the decay curves are shown. The subplots show the comparison for different natural signal decays (a) sample 148101 used to construct the MAAD curve with a natural dose of $45 \mathrm{~Gy}$, (b) sample 148110 with expected natural dose of $630 \mathrm{~Gy}$, (c) sample 148123 with an expected natural dose of $1700 \mathrm{~Gy}$, and (d) sample 148125 with an expected natural dose of 7900 Gy. Note that the MAAD decays curves are identical in all the four subplots.

\section{Figure 10}

(a) Comparison of natural (black filled circles) and MAAD (filled red triangles) DRC curves using early background subtraction. The data is reproduced here from Fig. 6, see details in the corresponding caption. (b) The equivalent doses, $\mathrm{D}_{\mathrm{e}}$ obtained using linear interpolation of the natural signals onto the regenerated DRC, are plotted as a function of the expected natural doses. Note that only 22 samples are shown in (b); sample 148101 has been omitted, as this sample was used to construct the MAAD DRC shown in (a) and cannot be interpolated onto itself. Furthermore, sample 148102 showed an anomalously low $\mathrm{L}_{\mathrm{n}} / \mathrm{T}_{\mathrm{n}}$ intensity, and could not be interpolated onto the MAAD DRC. 
Figure 11

Measured VSL ages from the Luochuan samples as a function of the expected ages provided by the independent chronology. The equivalent doses in Fig. 10b have been converted into VSL ages by using the dose rate for each of the 22 samples (Table 1). The grey shaded area corresponds to the region of natural VSL DRC saturation if assumed to be $2 \mathrm{D}_{0}=1840 \mathrm{~Gy}$. The black points (15 samples) correspond to the VSL ages in agreement within $10 \%$ of the independent ages, this is shown as a red 1:1 line with dashed lines corresponding to $\pm 10 \%$. Open grey symbols (7 samples) denote ages in disagreement with the independent chronology; note that 4 out of 7 disagreements occur beyond the estimated dating limit of VSL. 
Fig. 1
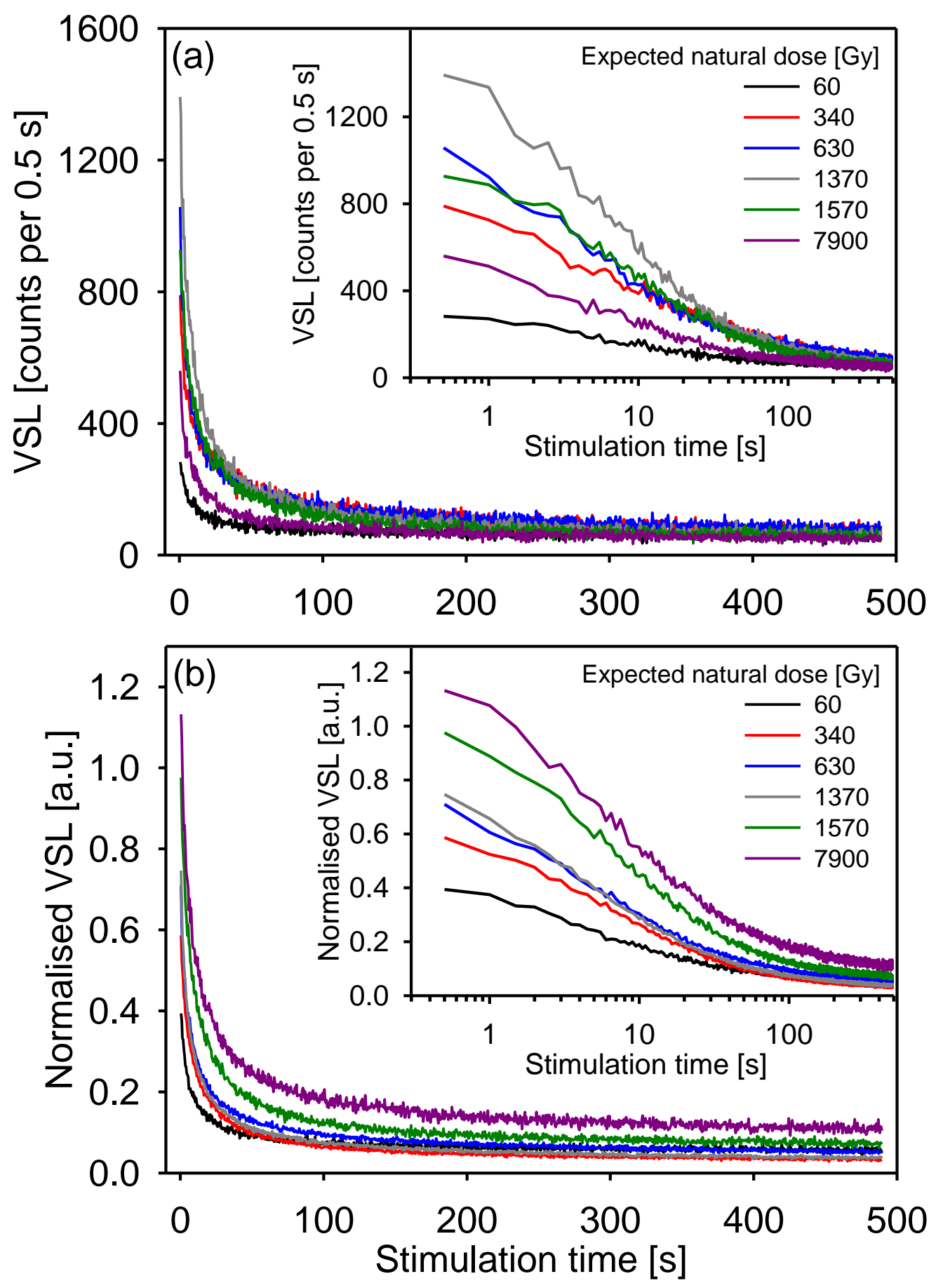
Fig. 2

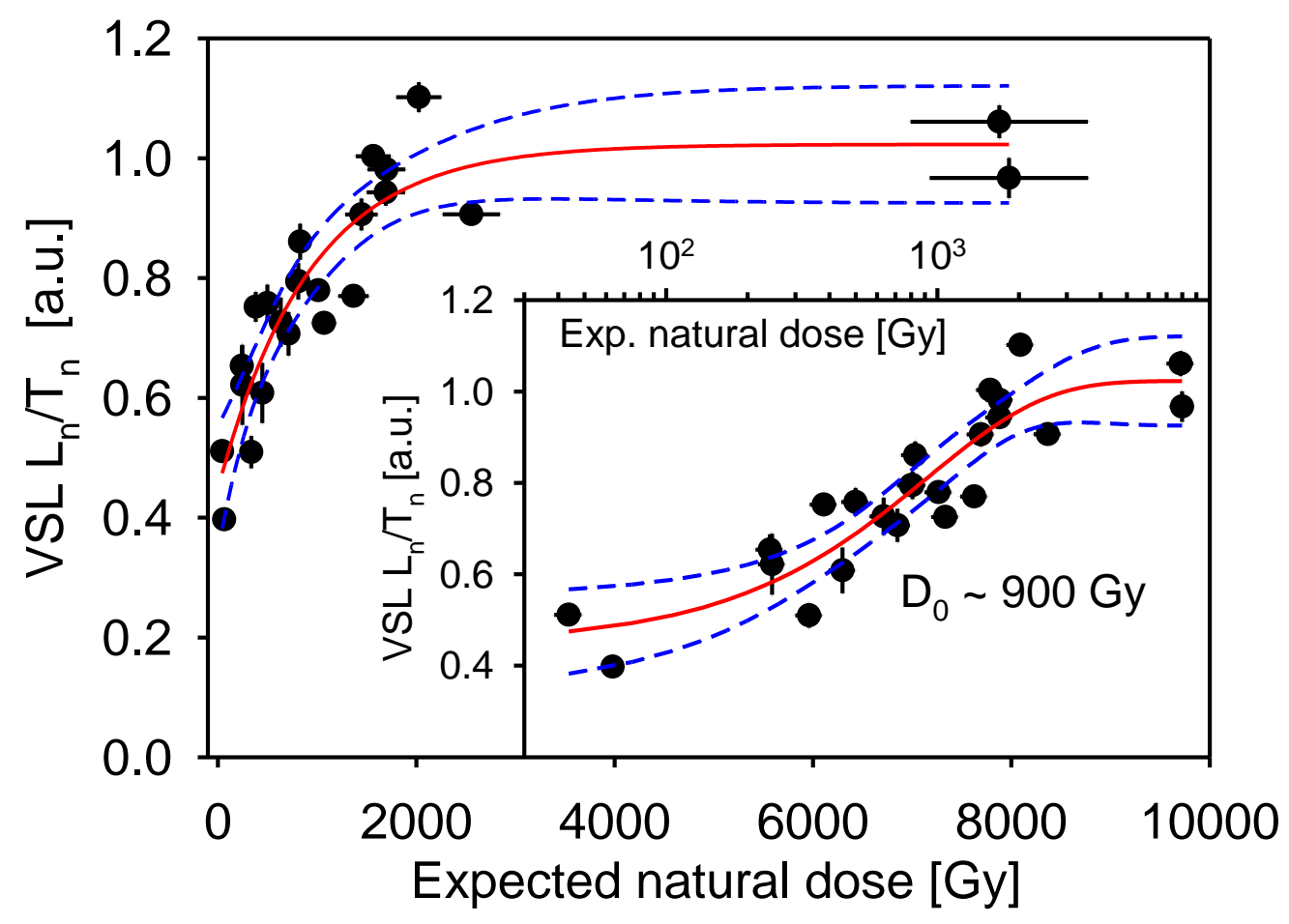


Fig. 3
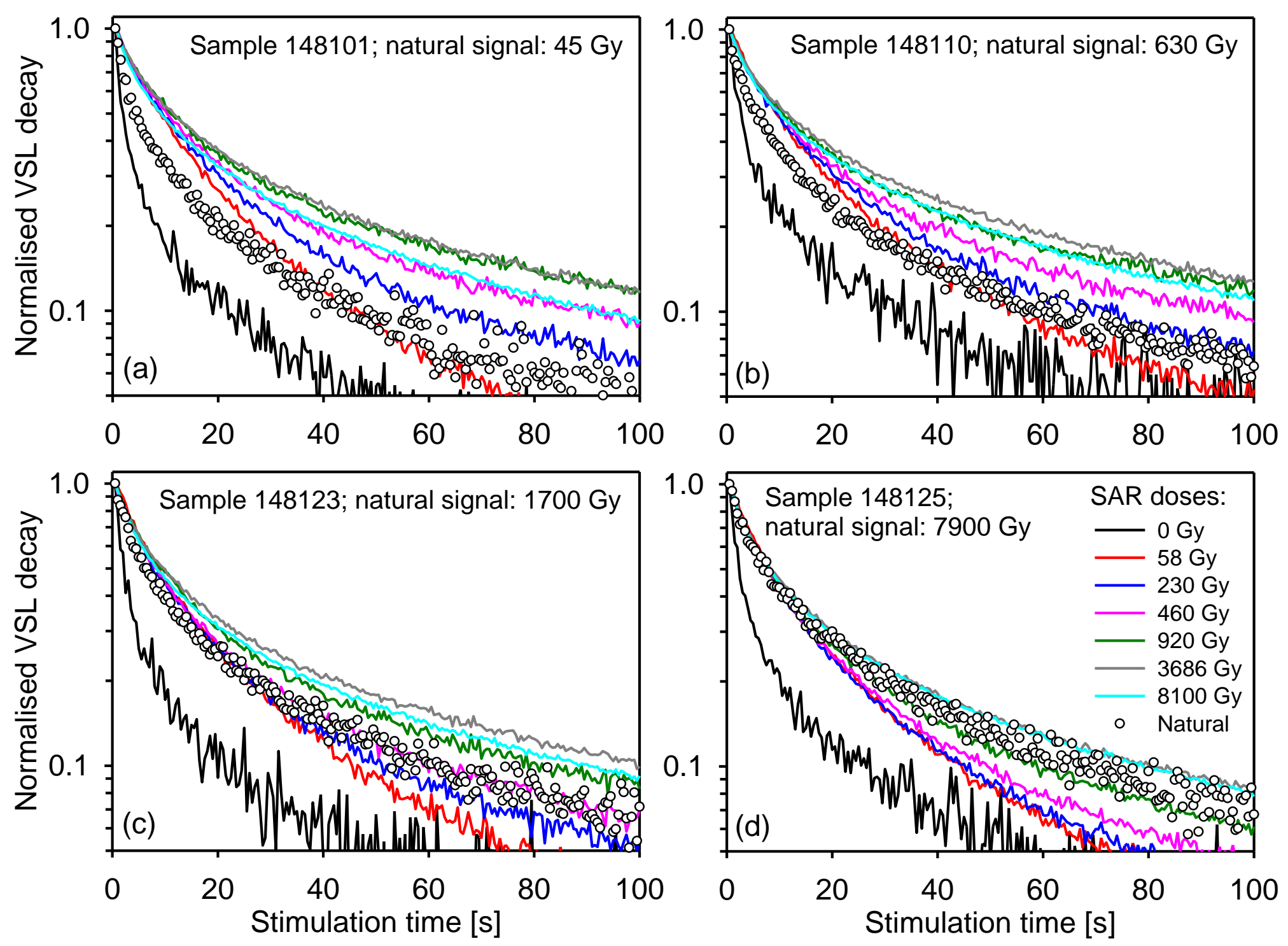
Fig. 4
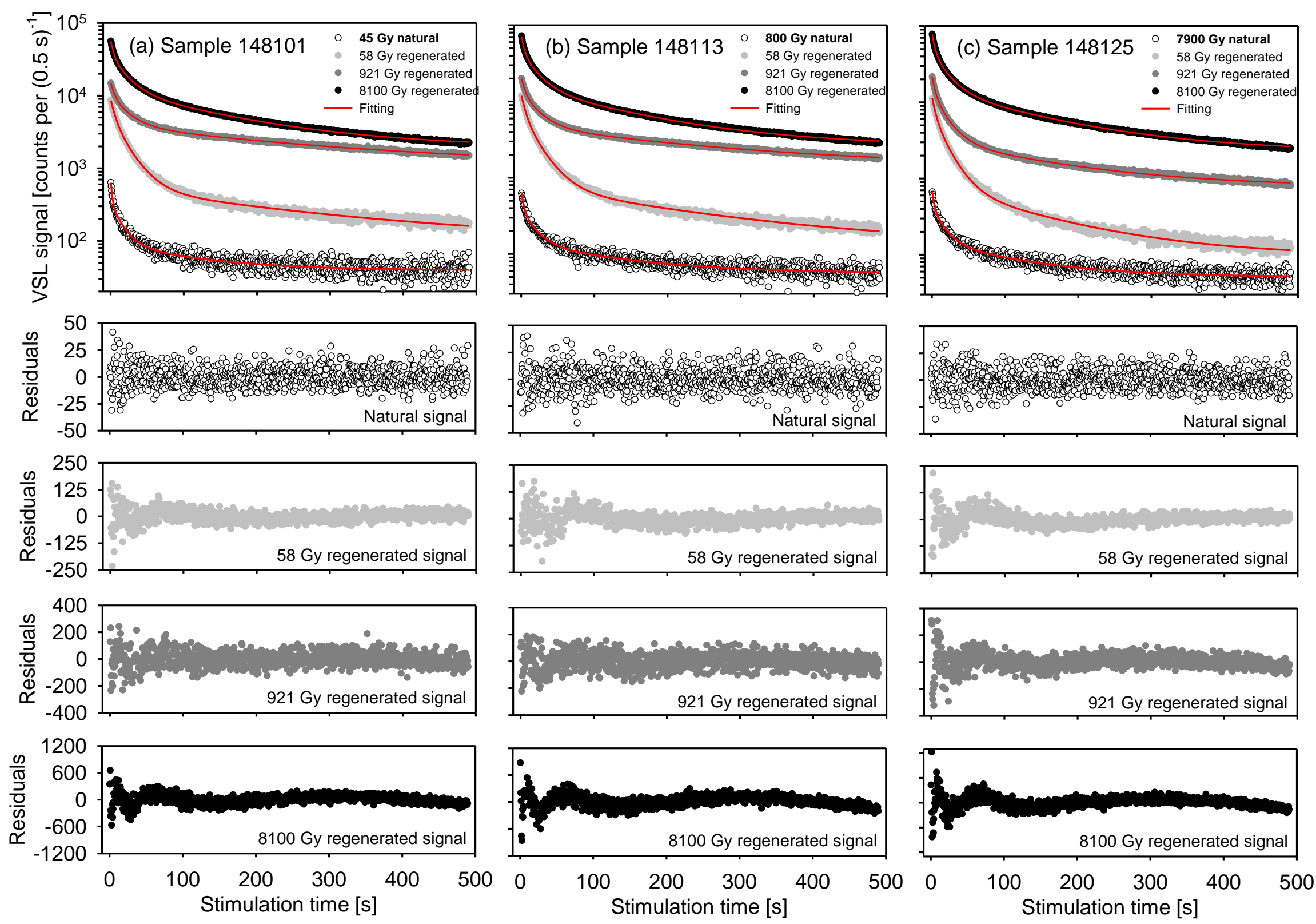
Fig. 5
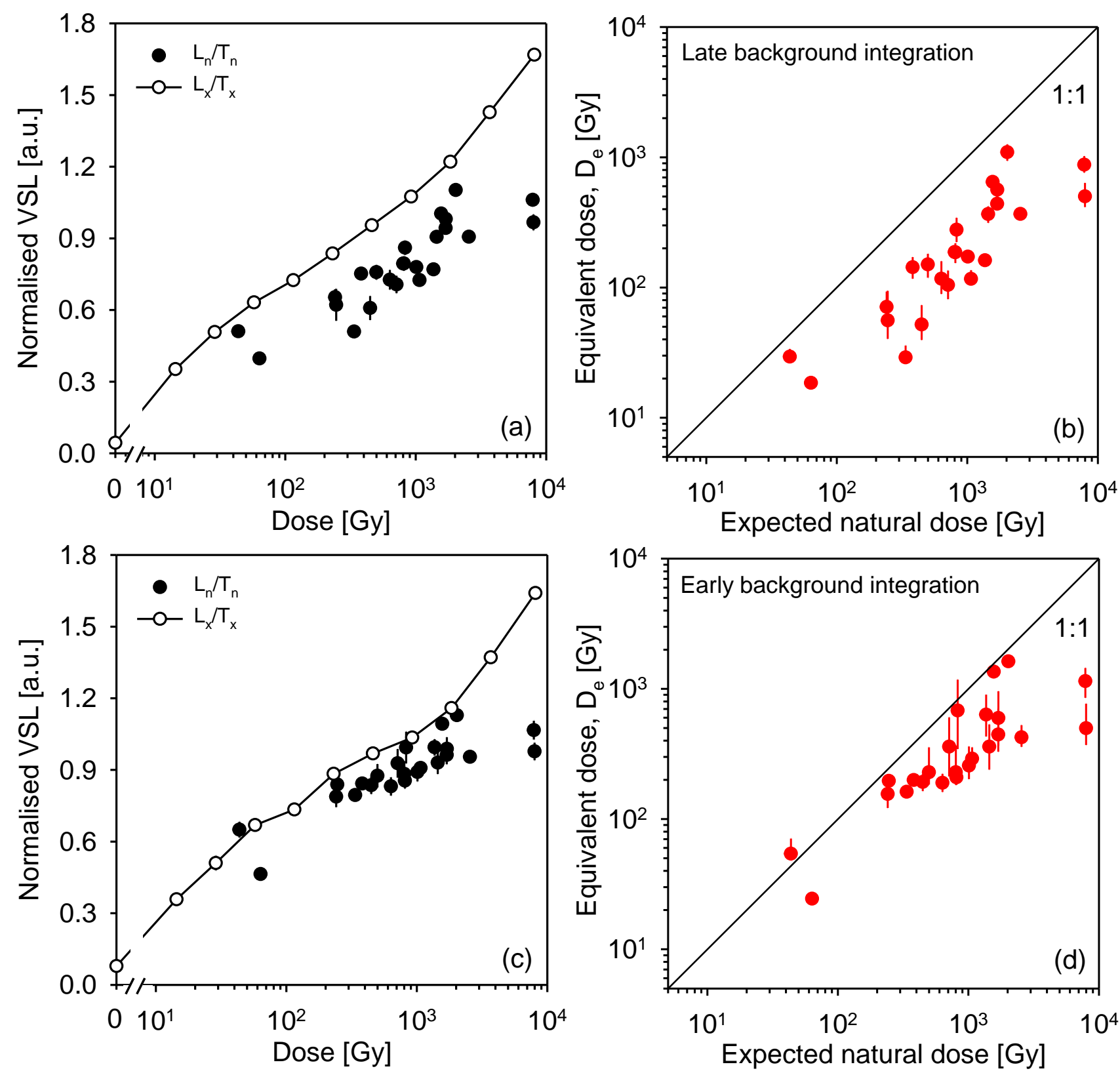
Fig. 6

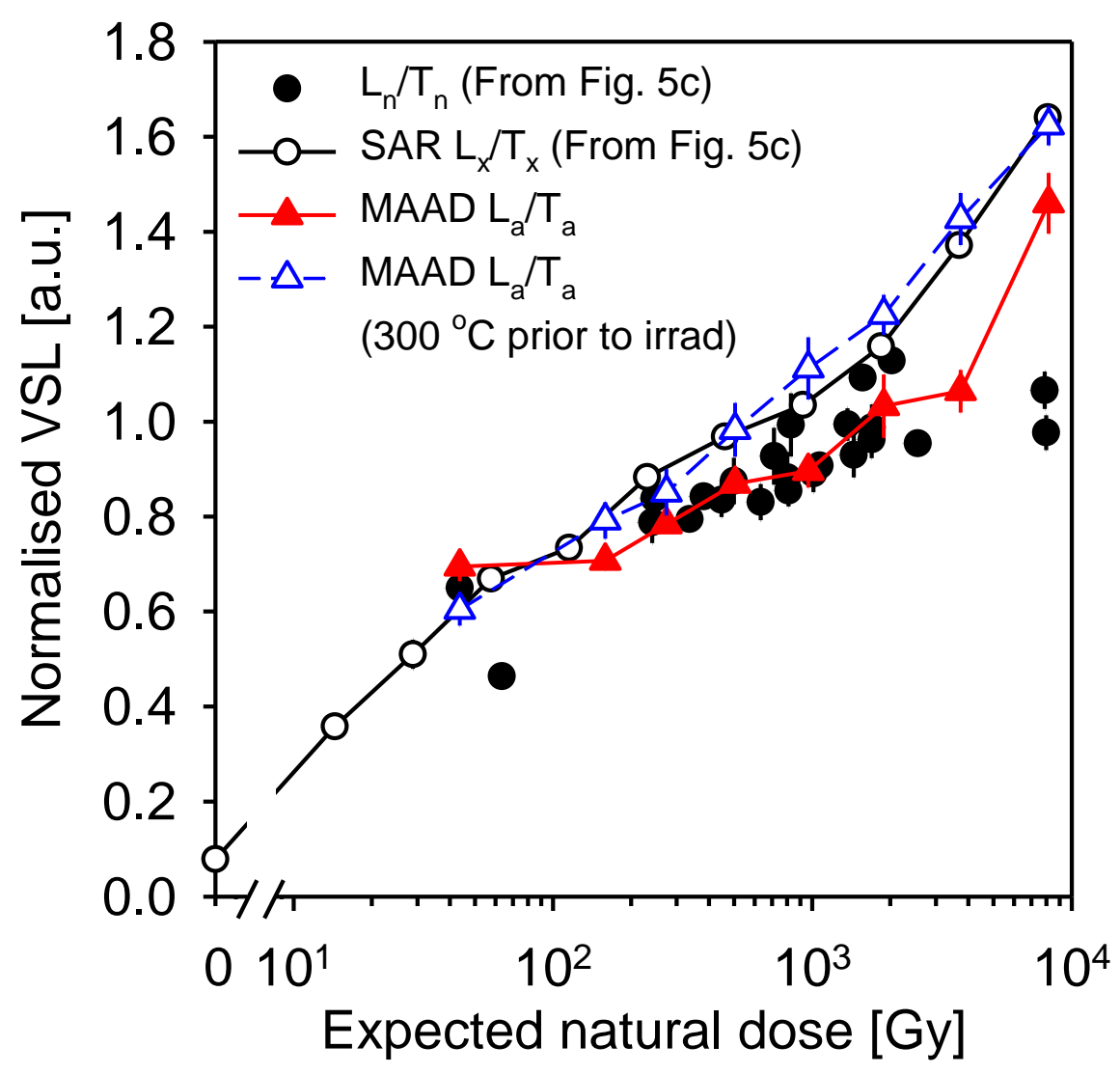


Fig. 7

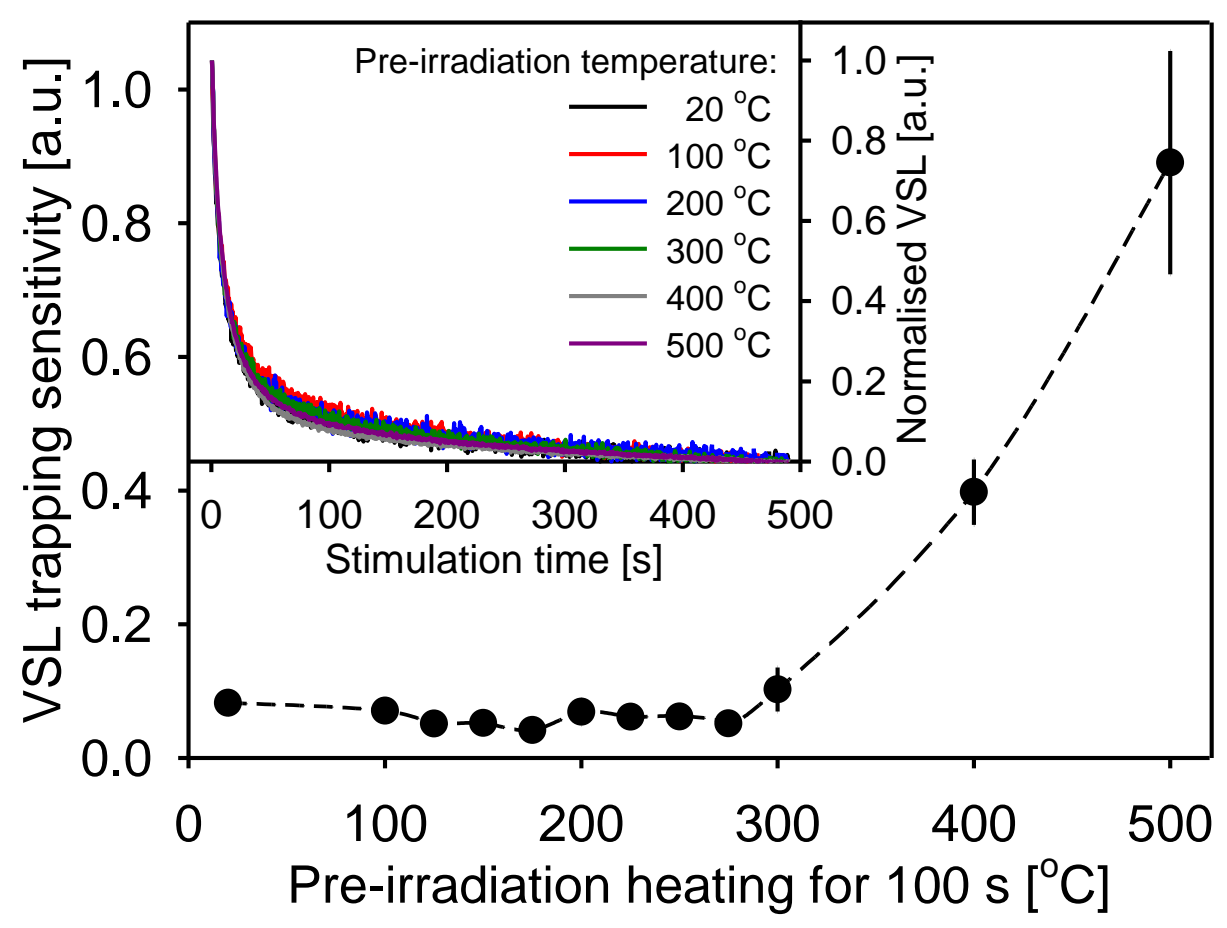


Fig. 8
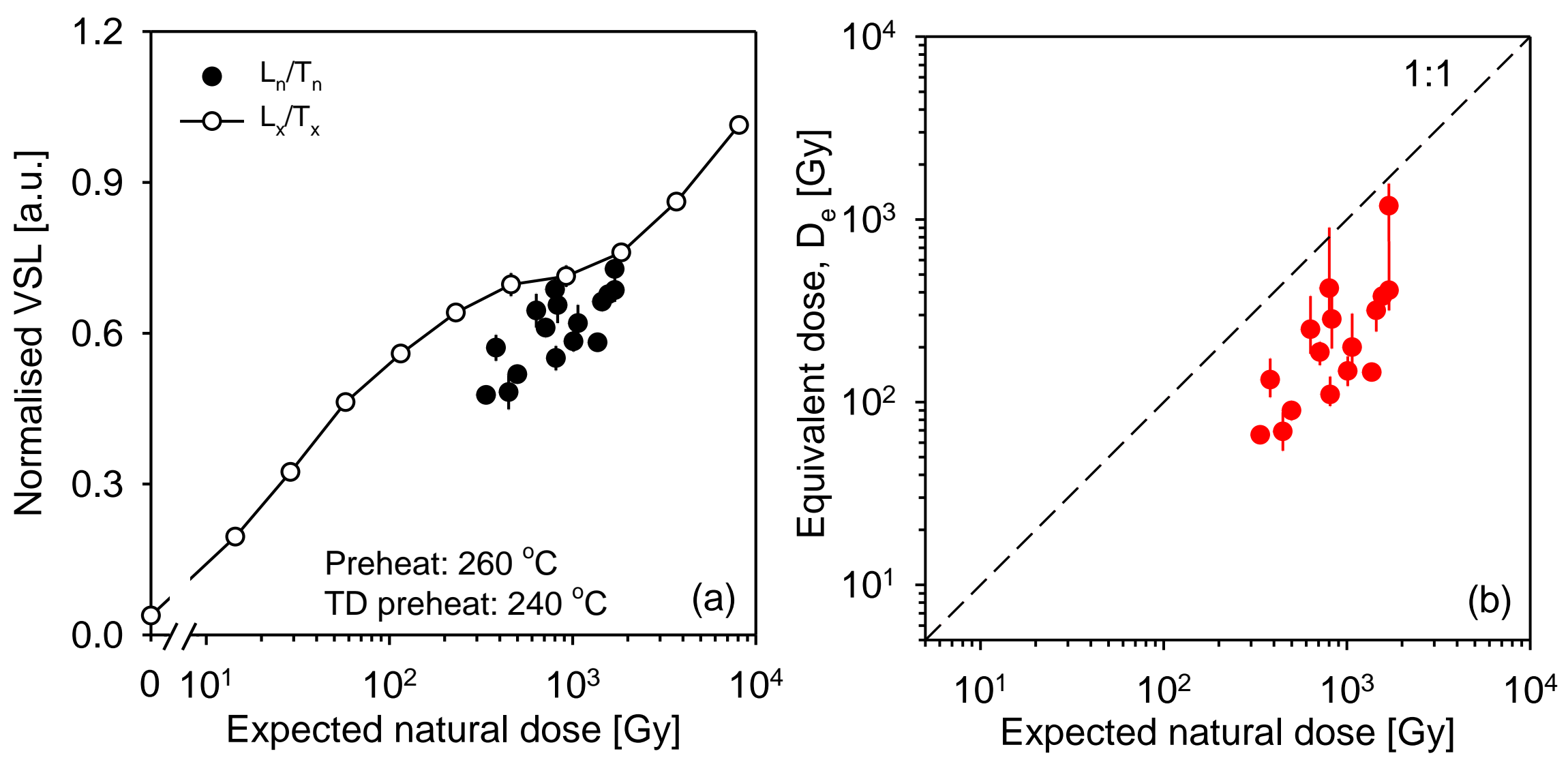
Fig. 9
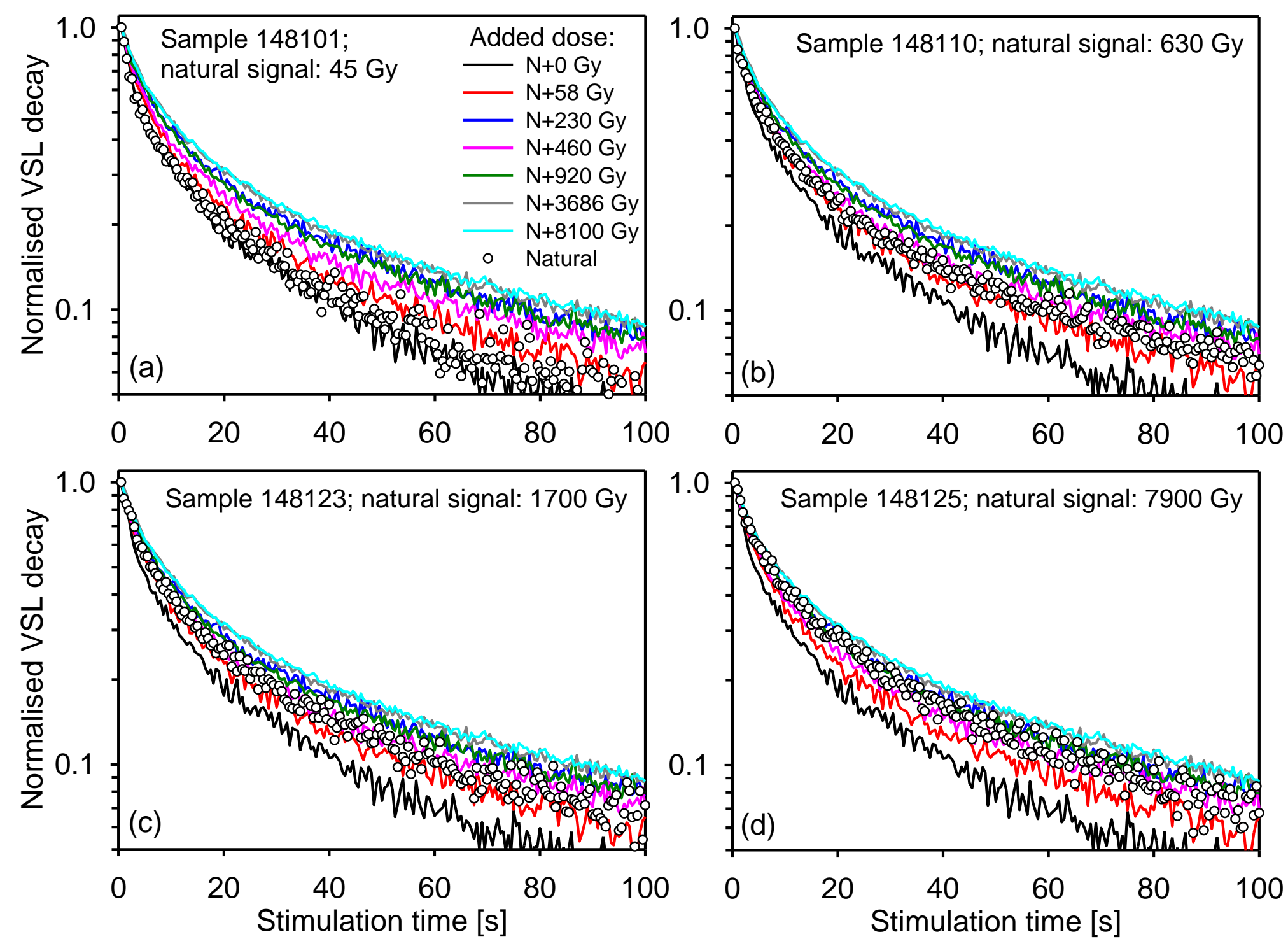
Fig. 10
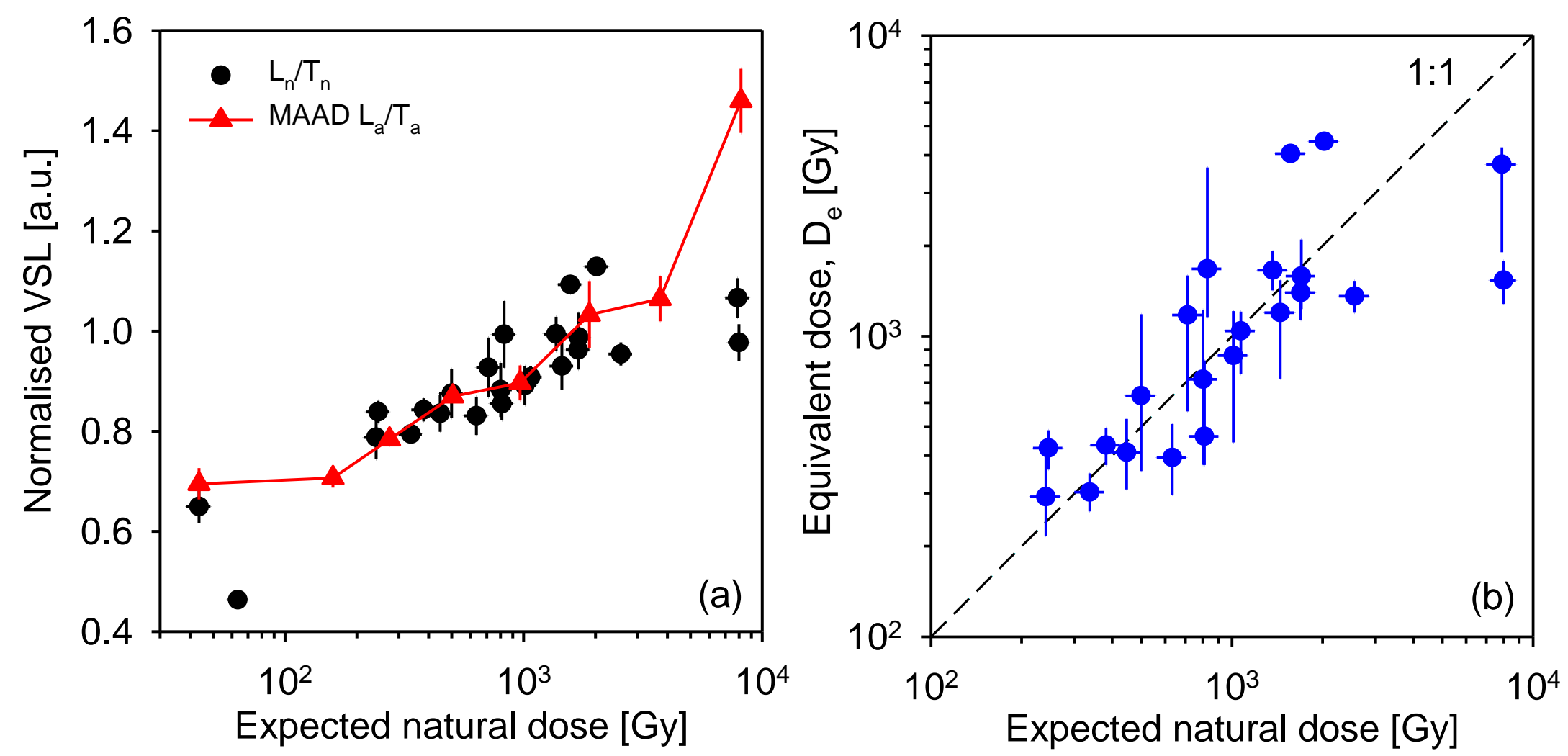
Fig. 11

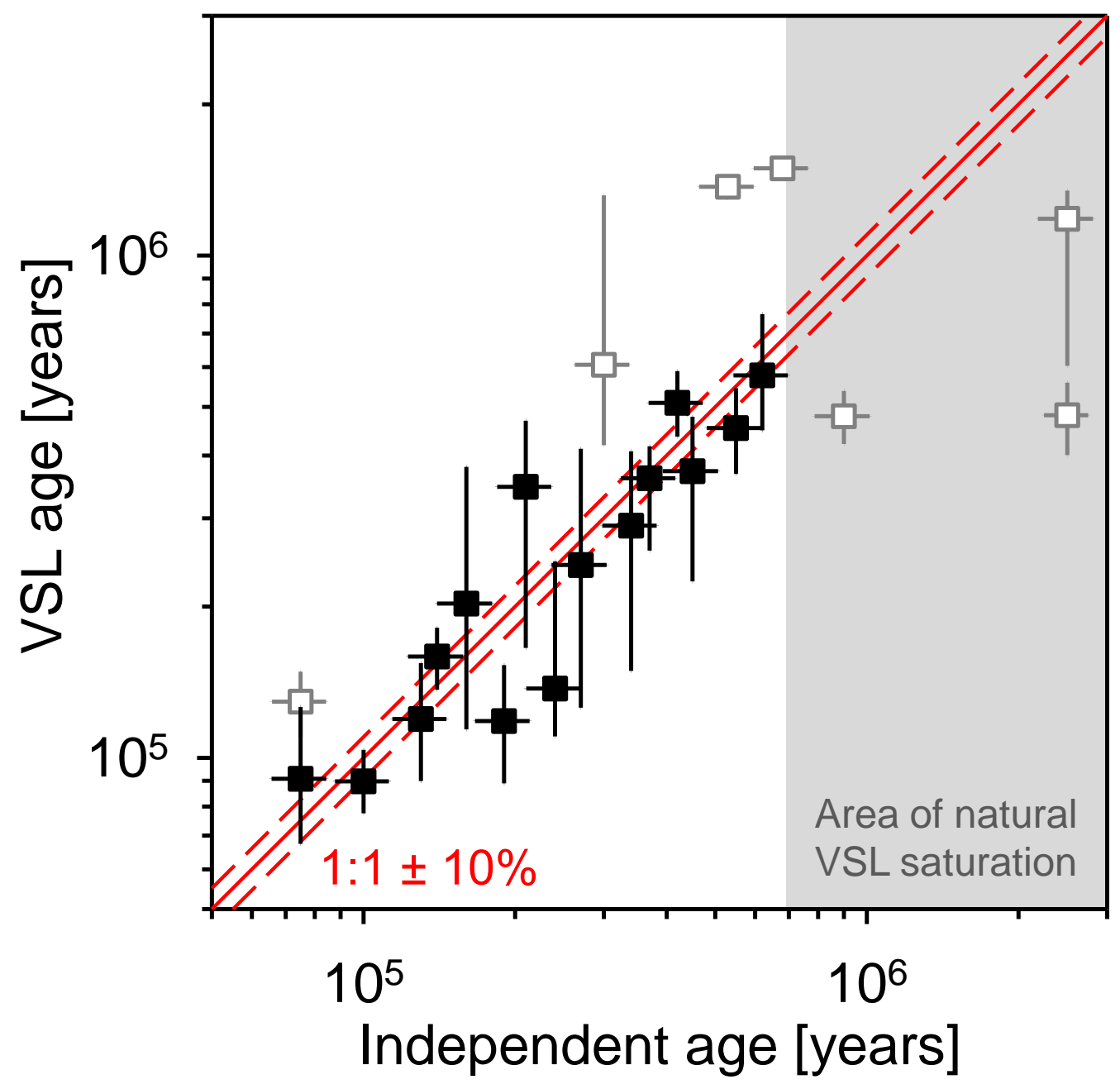


Table 1

\begin{tabular}{|c|c|c|c|c|c|c|c|c|c|c|c|c|}
\hline $\begin{array}{l}\text { Sample } \\
\text { code }\end{array}$ & Unit & $\begin{array}{l}\text { Depth, } \\
\text { m }\end{array}$ & $\begin{array}{c}\text { Expected age, } \\
\text { ka }\end{array}$ & $\begin{array}{l}{ }^{238} \mathrm{U} \\
\mathrm{Bq} / \mathrm{kg}\end{array}$ & $\begin{array}{l}{ }^{226} \mathrm{Ra}, \\
\mathrm{Bq} / \mathrm{kg}\end{array}$ & $\begin{array}{l}{ }^{232} \mathrm{Th}, \\
\mathrm{Bq} / \mathrm{kg}\end{array}$ & $\begin{array}{c}{ }^{40} \mathrm{~K}, \\
\mathrm{~Bq} / \mathrm{kg}\end{array}$ & $\begin{array}{c}\text { W.C.*, } \\
\%\end{array}$ & $\begin{array}{c}\text { Total dose rate, } \\
\text { Gy/ka }\end{array}$ & $\begin{array}{c}\text { Expected dose, } \\
\text { Gy }\end{array}$ & $\begin{array}{l}\text { MAAD VSL dose, } \\
\text { Gy }\end{array}$ & $\begin{array}{c}\text { MAAD VSL age, } \\
\text { ka }\end{array}$ \\
\hline 148101 & S0 & 0.5 & $11.2 \pm 1.2$ & $43 \pm 18$ & $47.0 \pm 1.5$ & $66.5 \pm 1.8$ & $809 \pm 25$ & 20 & $3.89 \pm 0.20$ & $44 \pm 5$ & - & - \\
\hline 148102 & L1-top & 1.0 & $19.7 \pm 1.6$ & $37 \pm 9$ & $37.7 \pm 0.8$ & $52.0 \pm 1.0$ & $625 \pm 14$ & 15 & $3.21 \pm 0.16$ & $63 \pm 6$ & - & - \\
\hline 148104 & L1-bottom & 10.0 & $75 \pm 8$ & $60 \pm 11$ & $40.7 \pm 1.0$ & $55.4 \pm 1.0$ & $604 \pm 17$ & 15 & $3.21 \pm 0.17$ & $241 \pm 27$ & $292_{-75}^{+114}$ & $91_{-23}^{+35}$ \\
\hline 148105 & S1-top & 10.5 & $75 \pm 8$ & $36 \pm 11$ & $39.9 \pm 1.0$ & $59.2 \pm 1.2$ & $702 \pm 19$ & 20 & $3.28 \pm 0.17$ & $246 \pm 28$ & $424_{-65}^{+62}$ & $129_{-20}^{+19}$ \\
\hline 148106 & S1-middle & 11.3 & $100 \pm 10$ & $40 \pm 11$ & $38.6 \pm 0.9$ & $61.1 \pm 1.2$ & $729 \pm 17$ & 20 & $3.37 \pm 0.17$ & $337 \pm 38$ & $303_{-41}^{+47}$ & $90_{-12}^{+14}$ \\
\hline 148107 & S1-bottom & 12.0 & $130 \pm 13$ & $51 \pm 11$ & $45.4 \pm 1.0$ & $65.8 \pm 1.0$ & $691 \pm 17$ & 20 & $3.44 \pm 0.17$ & $447 \pm 50$ & $411_{-102}^{+120}$ & $119_{-30}^{+35}$ \\
\hline 148108 & L2-top & 15.0 & $140 \pm 14$ & $33 \pm 10$ & $32.3 \pm 0.9$ & $47.3 \pm 1.1$ & $545 \pm 16$ & 15 & $2.73 \pm 0.15$ & $382 \pm 43$ & $433_{-61}^{+61}$ & $159_{-22}^{+22}$ \\
\hline 148109 & L2-middle & 17.0 & $160 \pm 16$ & $50 \pm 12$ & $35.0 \pm 1.0$ & $53.3 \pm 1.2$ & $628 \pm 17$ & 15 & $3.12 \pm 0.17$ & $499 \pm 57$ & $633_{-277}^{+552}$ & $203_{-89}^{+177}$ \\
\hline 148110 & S2-top & 18.0 & $190 \pm 19$ & $70 \pm 14$ & $38.2 \pm 1.2$ & $65.8 \pm 1.2$ & $664 \pm 21$ & 20 & $3.33 \pm 0.17$ & $634 \pm 71$ & $394_{-98}^{+116}$ & $118_{-29}^{+35}$ \\
\hline 148111 & S2-middle & 19.5 & $210 \pm 21$ & $64 \pm 11$ & $39.6 \pm 0.9$ & $63.7 \pm 0.9$ & $702 \pm 17$ & 20 & $3.39 \pm 0.17$ & $712 \pm 80$ & $1177_{-615}^{+415}$ & $347_{-181}^{+122}$ \\
\hline 148112 & S2-bottom & 21.0 & $240 \pm 24$ & $49 \pm 12$ & $39.5 \pm 1.0$ & $65.2 \pm 1.2$ & $708 \pm 17$ & 20 & $3.38 \pm 0.17$ & $810 \pm 91$ & $464_{-91}^{+366}$ & $137_{-27}^{+109}$ \\
\hline 148113 & L3-middle & 23.0 & $270 \pm 27$ & $28 \pm 11$ & $35.0 \pm 0.9$ & $53.0 \pm 1.1$ & $610 \pm 16$ & 15 & $2.97 \pm 0.16$ & $801 \pm 91$ & $718_{-345}^{+506}$ & $242_{-116}^{+171}$ \\
\hline 148114 & S3-top & 24.0 & $300 \pm 30$ & $27 \pm 11$ & $33.5 \pm 1.0$ & $52.7 \pm 1.2$ & $596 \pm 18$ & 20 & $2.76 \pm 0.14$ & $828 \pm 93$ & $1674_{-517}^{+1968}$ & $606_{-187}^{+713}$ \\
\hline 148115 & S3-bottom & 26.0 & $340 \pm 34$ & $58 \pm 12$ & $33.6 \pm 1.0$ & $54.8 \pm 1.0$ & $625 \pm 18$ & 20 & $2.97 \pm 0.15$ & $1011 \pm 114$ & $862_{-420}^{+350}$ & $290_{-141}^{+118}$ \\
\hline 148117 & S4-top & 31.0 & $370 \pm 37$ & $23 \pm 11$ & $35.1 \pm 1.0$ & $55.3 \pm 1.2$ & $633 \pm 19$ & 20 & $2.89 \pm 0.15$ & $1069 \pm 121$ & $1042_{-294}^{+165}$ & $360_{-102}^{+57}$ \\
\hline 148118 & S4-bottom & 33.0 & $420 \pm 42$ & $41 \pm 12$ & $32.3 \pm 1.0$ & $61.5 \pm 1.3$ & $726 \pm 19$ & 20 & $3.25 \pm 0.17$ & $1367 \pm 154$ & $1659_{-238}^{+258}$ & $510_{-73}^{+79}$ \\
\hline 148119 & L5-middle & 36.0 & $450 \pm 45$ & $60 \pm 12$ & $38.0 \pm 1.0$ & $54.8 \pm 1.0$ & $640 \pm 18$ & 15 & $3.21 \pm 0.17$ & $1446 \pm 164$ & $1197_{-476}^{+338}$ & $373_{-148}^{+105}$ \\
\hline 148121 & S5-bottom & 43.0 & $530 \pm 53$ & $35 \pm 12$ & $31.0 \pm 1.0$ & $55.8 \pm 1.3$ & $655 \pm 19$ & 20 & $2.95 \pm 0.15$ & $1566 \pm 176$ & $4051_{-205}^{+207}$ & $1371_{-69}^{+70}$ \\
\hline 148122 & L6-middle & 45.5 & $550 \pm 55$ & $52 \pm 11$ & $36.0 \pm 0.9$ & $51.8 \pm 0.9$ & $626 \pm 17$ & 15 & $3.08 \pm 0.17$ & $1693 \pm 192$ & $1397_{-265}^{+281}$ & $454_{-86}^{+91}$ \\
\hline 148123 & S6-top & 46.3 & $620 \pm 62$ & $35 \pm 10$ & $30.8 \pm 0.9$ & $50.9 \pm 1.1$ & $560 \pm 17$ & 20 & $2.74 \pm 0.14$ & $1700 \pm 191$ & $1583_{-353}^{+515}$ & $578_{-129}^{+188}$ \\
\hline 148124 & S6-bottom & 60.0 & $680 \pm 68$ & $28 \pm 12$ & $34.1 \pm 1.0$ & $56.9 \pm 1.3$ & $656 \pm 17$ & 20 & $2.98 \pm 0.15$ & $2025 \pm 228$ & $4444_{-143}^{+152}$ & $1492_{-48}^{+51}$ \\
\hline 128187 & L9-middle & 60.0 & $900 \pm 90$ & $36 \pm 9$ & $31.7 \pm 0.8$ & $49.1 \pm 1.0$ & $592 \pm 14$ & 15 & $2.84 \pm 0.15$ & $2555 \pm 290$ & $1536_{-257}^{+247}$ & $479_{-57}^{+59}$ \\
\hline 148125 & Loess-Red Clay boundary & 130.0 & $2500 \pm 250$ & $44 \pm 12$ & $31.4 \pm 1.0$ & $56.3 \pm 1.2$ & $656 \pm 17$ & 15 & $3.15 \pm 0.17$ & $7879 \pm 895$ & $1360_{-162}^{+167}$ & $481_{-80}^{+77}$ \\
\hline 128188 & Loess-Red Clay boundary & 130.0 & $2500 \pm 250$ & $68 \pm 13$ & $35.5 \pm 1.2$ & $54.7 \pm 1.2$ & $366 \pm 21$ & 15 & $3.19 \pm 0.02$ & $7977 \pm 800$ & $3732_{-1829}^{+516}$ & $1184_{-580}^{+164}$ \\
\hline
\end{tabular}


Table 2

\begin{tabular}{|c|l|}
\hline Step & VSL protocol \\
\hline 1 & Irradiation $(\mathrm{x} \mathrm{Gy})$ \\
2 & Preheat $\left(300{ }^{\circ} \mathrm{C}, 100 \mathrm{~s}\right)$ \\
3 & Blue bleach $\left(125{ }^{\circ} \mathrm{C}, 100 \mathrm{~s}\right)$ \\
4 & VSL $\left(30{ }^{\circ} \mathrm{C}, 500 \mathrm{~s}\right) \rightarrow \mathrm{L}_{\mathrm{n}}$ or $\mathrm{L}_{\mathrm{x}}\left(\right.$ or $\left.\mathrm{L}_{\mathrm{a}}\right)$ \\
5 & Irradiation $(540 \mathrm{~Gy})$ \\
6 & Preheat $\left(300{ }^{\circ} \mathrm{C}, 100 \mathrm{~s}\right)$ \\
7 & Blue bleach $\left(125{ }^{\circ} \mathrm{C}, 100 \mathrm{~s}\right)$ \\
8 & VSL $\left(30{ }^{\circ} \mathrm{C}, 500 \mathrm{~s}\right) \rightarrow \mathrm{T}_{\mathrm{n}}$ or $\mathrm{T}_{\mathrm{x}}\left(\right.$ or $\left.\mathrm{T}_{\mathrm{a}}\right)$ \\
9 & TL to $500{ }^{\circ} \mathrm{C}$ for $20 \mathrm{~s}$ \\
10 & For SAR, repeat steps $1-9$ \\
\hline
\end{tabular}

